\title{
MEAN FIELD DYNAMICS OF COLLISIONAL PROCESSES WITH DUPLICATION, LOSS AND COPY
}

\author{
FEDERICO BASSETTI, GIUSEPPE TOSCANI
}

\begin{abstract}
In this paper we introduce and discuss kinetic equations for the evolution of the probability distribution of the number of particles in a population subject to binary interactions. The microscopic binary law of interaction is assumed to be dependent on fixed-in-time random parameters which describe both birth and death of particles, and the migration rule. These assumptions lead to a Boltzmann-type equation that in the case in which the mean number of the population is preserved, can be fully studied, by obtaining in some case the analytic description of the steady profile. In all cases, however, a simpler kinetic description can be derived, by considering the limit of quasi-invariant interactions. This procedure allows to describe the evolution process in terms of a linear kinetic transport-type equation. Among the various processes that can be described in this way, one recognizes the Lea-Coulson model of mutation processes in bacteria, a variation of the original model proposed by Luria and Delbrück.
\end{abstract}

\section{INTRODUCTION}

The description of emerging collective behaviors and self-organization in multi-agent interactions started to gain popularity in the recent years and it represents one of the major challenges in contemporary mathematical modeling. In the biological context, the emergent behavior of bird flocks, fish schools or bacteria aggregations, among others, is a major research topic in population and behavioral biology and ecology [19, 20, 22, 23, 24, 32]. Other important examples of emergent behaviors describe building of tumors by cancer cells and their migration through the tissues $[8,9,10,11,33]$. Another famous example to consider in this contest is the classical LuriaDelbrück mutation problem $[36,38,39]$. Nonlinear statistical physics represents a powerful tool to describe these different biological phenomena. In particular, methods borrowed from kinetic theory of rarefied gases have been successfully employed to construct master equations of Boltzmann type, usually referred to as kinetic equations, describing the time-evolution of the number density of the population and, eventually, the emergence of universal behaviors through their equilibria $[41,43]$.

The building block of kinetic theory is represented by binary interactions, which, similarly to binary interactions between particles velocities in the classical kinetic theory of rarefied gases, describe the variation law of some selected agent characteristic, like its number. Then, the microscopic law of variation of the number density consequent to the (fixed-in-time) way of interaction, is able to capture both the time evolution and the steady profile, in presence of some conservation law $[41,43]$.

In this paper, we are interested in studying processes in which a huge population of $N$ interacting agents can be characterized in terms of some scalar quantity assuming integer non-negative values, say $\left(V_{1}, \ldots, V_{N}\right)$. The binary interactions between agents are described by the following rule. When two agents $i$ and $j$ interact, their pre-interaction values $\left(V_{i}, V_{j}\right)$ change to

$$
V_{i}^{\prime}=\sum_{k=1}^{V_{i}} X_{i k}+\sum_{k=1}^{V_{j}} Y_{i k}, \quad V_{j}^{\prime}=\sum_{k=1}^{V_{j}} X_{j k}+\sum_{k=1}^{V_{i}} Y_{j k} .
$$

In (1), the quantities $X_{i k}, Y_{i k}, X_{j k}, Y_{j k}$ are independent random variables which assume nonnegative integer values, the $X$ 's with density function $p_{X}$ and the $Y$ 's with density function $p_{Y}$.

In [31], (Case 1), a collisional process of type (1), with $X_{j k} \in\{0,1,2\}$ and $Y_{j k} \in\{0,1\}$, has been introduced to describe the evolution of the gene-family abundance (the number of genes of a given family found in a genome) trough a minimal dynamics of duplication, loss and interspecies 
horizontal gene transfer (HGT) According to this model, a fixed number $N$ of species-genomes interact pairwise randomly, so that a given family can gain genes by interactions associated with HGT events. When two species interact they can exchange genes by HGT by drawing them from each other with Bernoulli trials of probability $p_{h}$. In the same time, they draw from their own genome genes to be lost (with probability $p_{l}$ ) and duplicated (with probability $p_{d}$ ). In summary, in this case $P\left\{X_{i k}=0\right\}=p_{l}, P\left\{X_{i k}=2\right\}=p_{d}, P\left\{X_{i k}=1\right\}=1-p_{l}-p_{d}, P\left\{Y_{i k}=1\right\}=$ $1-P\left\{Y_{i k}=0\right\}=p_{h}$. Note that, by taking $p_{d}+p_{h}=p_{l}$, the mean number of genes in a given family is conserved in each collision.

More generally, binary interactions (1) can be seen as a process in which the $N$ agents can vary their given quantity $V_{i}$ of some objects (the genes in Case 1) according to a collisional processes with duplication, loss and copy. An alternative interpretation of the process relies in considering populations in place of individuals, and individuals in place of objects. In this case, populations evolve following a classical branching process, and in addition interact with the other branching populations and exchange "migrants". Note that, within this interpretation, the $k$-th individual of the population $i$, interacting with population $j$, has $X_{i k}$ children that remain in the population $i$ (do not migrate) and $Y_{j k}$ that migrate to population $j$.

An example of this second interpretation (Case 2) is a process in which $N$ populations of cells interact and are subject to a mutation process. Here $V_{j}$ represent the number of mutant cells in the $j$ population. Each mutating cell can produce a clone in the same population with probability $p$ and, independently, it can produce a clone which "migrates" to the other population with probability $q$. In other words $P\left\{X_{i k}=2\right\}=p$, and $P\left\{X_{i k}=1\right\}=1-p$ and $P\left\{Y_{i k}=\right.$ $1\}=1-P\left\{Y_{i k}=0\right\}=q$. Here typically one consider situation in which the mean number of mutants grown in time. As we shall see this process of growth is similar to the linear process for cells mutation proposed by Lea and Coulson [36], which has its origin in a series of pioneering experiments proposed by Luria and Delbrück [39].

Our aim here is to make use of classical methods of kinetic theory to provide a kinetic description of the evolution in time of a multi-agent system obeying to binary interactions of type (1). Making use of this collisional mechanism between individuals, we introduce a bilinear Boltzmann-type equation which describes the behavior of the population in terms of its density $f(v, t)$, where $v$ represents the number of objects. Next, in the asymptotic procedure usually referred to as grazing collision limit, we obtain a simpler linear equation in divergence form, which retains many properties of the underlying Boltzmann equation, and in addition can be studied in detail.

Having in mind as prototypes both the horizontal gene transfer model in [31] (Case 1), and the variant of Luria-Delbrück mutation model in [33, 47] (Case 2), we will split our analysis in two sub-cases, identified by the evolution of the mean density. Thus, we will limit our study to the cases in which the mean density of the population remains constant, or grows in time. In both cases, we can identify in a precise way the large time behavior of the solution. In particular, in presence of conservation of the mean number of objects (Case 1), it will be shown that the solution density converges to a steady state profile, which depends heavily from the microscopic interactions.

In more details, in Section 2 we will briefly introduce the kinetic description of our collisional models, coupling them with some direct physical consequences. This allows the interested reader to take an exhaustive view of the modeling assumptions, by comparing both the nonlinear Boltzmanntype equation with its linear asymptotics, named quasi-invariant limit. Also, the main differences between Cases 1 and 2 are here underlined. Next, we collect in Section 3 the main results on existence and uniqueness of solutions to the mathematical models, by computing additionally some of the relevant mean quantities. Here, convergence to equilibrium for collisions of type (1), which imply conservation of the mean, value is studied in full details. Section 4 will deal with the large-time behaviour of the solution in the case in which collisions of type (1) imply the growth of the mean value. In the case of growth, by suitably scaling the solution with respect to its time-dependent mean value we will show that the scaled solution converges towards a fixed steady profile as time tends towards infinity. Last, Section 5 will describe and justify from a mathematical point of view the quasi-invariant asymptotics procedure leading from the Boltzmann-type equation to the linear one. Some technical results are for the sake of readability postponed to the Appendix. 


\section{ThE KINETIC EQUATION AND ITS QUASI-INVARIANT COLLISION LIMIT}

2.1. The kinetic equation. Performing the usual mean-field approximation when the number of particles (colliding entities) goes to infinity, the evolution in time of the number density can be quantitatively described by a bilinear Boltzmann-type equation. In this equation, the time variation of the density $f_{t}(v)=f(v, t)$, with $v \in \mathbb{N}$ and $t>0$, follows from a balance between gain and loss terms, that, for the given number $v$, take into account all the interactions of type (1) which end up with the number $v$ (gain term) as well as all the interactions which, starting from the number $v$, lose this value after interaction (loss term). This Boltzmann-type equation reads [43]

$$
\frac{\partial}{\partial t} f_{t}(v)=Q^{+}\left(f_{t}, f_{t}\right)(v)-f_{t}(v)
$$

In (2), $Q^{+}$is the gain collision operator defined, for any pair of densities $f$ and $g$ on $\mathbb{N}$, by

$$
Q^{+}(f, g)(v)=\operatorname{Prob}\left(\sum_{i=1}^{V_{1}} Y_{i}+\sum_{i=1}^{V_{2}} X_{i}=v\right),
$$

where $V_{1}, V_{2}, X_{1}, X_{2}, \ldots, Y_{1}, Y_{2} \ldots$ are stochastically independent, $V_{1}$ has density $f, V_{2}$ has density $g$, the $X_{i}$ 's have the same law of a random variable $X$ with density $p_{X}$ and the $Y_{i}$ 's have the same law of a random variable $Y$ with density $p_{Y}$.

Equation (2) is coupled with an initial condition $f_{0}(v)$, which is here assumed to be a probability density on $\mathbb{N}$. Considering that the variable $v$ can only assume values in $\mathbb{N}$, equation (2) can be fruitfully rewritten in terms of probability generating functions (p.g.f.)

$$
\hat{f}_{t}(z):=\sum_{v \geq 0} z^{v} f_{t}(v) \quad z \in[0,1] .
$$

A standard computation shows that $\hat{f}_{t}(z)$ satisfies the (simpler) nonlinear equation

$$
\begin{aligned}
& \frac{\partial}{\partial t} \hat{f}_{t}(z)=\hat{f}_{t}\left(\hat{p}_{X}(z)\right) \hat{f}_{t}\left(\hat{p}_{Y}(z)\right)-\hat{f}_{t}(z) \quad z \in[0,1], t>0, \\
& \hat{f}_{0}(z)=\sum_{v \geq 0} z^{v} f_{0}(v)
\end{aligned}
$$

where

$$
\hat{p}_{X}(z)=\mathbb{E}\left[z^{X}\right]=\sum_{m \geq 0} z^{m} p_{X}(m) \quad \text { and } \quad \hat{p}_{Y}(z)=\mathbb{E}\left[z^{Y}\right]=\sum_{m \geq 1} z^{m} p_{Y}(m) .
$$

Equation (4) is the analogous of the Fourier transformed Boltzmann equation for maxwell pseudomolecules, introduced in kinetic theory of rarefied gases by Bobylev [13].

To avoid trivial situations, in what follows we shall assume that both $P\{X=0\} \neq 1$ and $P\{Y=0\} \neq 1$.

Existence and uniqueness of solution of equation (4) can be proven in a standard way (cf. Section 3.1 for details).

In this paper, we will only consider random variables $X$ and $Y$ such that

$$
\mathbb{E}\left[X^{r}+Y^{r}\right]<+\infty
$$

for some $r \geq 1$. In presence of condition (5) for some integer $r \geq 1$, and assuming that the initial density $f_{0}(v)$ has bounded moments up to order $r$, one can easily reckon explicit expressions for the evolution of the moments of $f_{t}(v)$. In particular, the mean $M_{1}\left(f_{t}\right)=\sum_{v} v f_{t}(v)$ of $f_{t}$ evolves according to

$$
M_{1}\left(f_{t}\right)=M_{1}\left(f_{0}\right) e^{\alpha_{1} t},
$$

where $\alpha_{1}:=\mathbb{E}[(X+Y)]-1$. Indeed, recall that for any p.g.f. $\hat{\rho}$ (of a probability $\rho$ on $\mathbb{N}$ )

$$
M_{1}(\rho):=\sum_{v} v \rho(v)=\left.\partial_{z} \hat{\rho}(z)\right|_{z=1},
$$


where $\left.\partial_{z} \hat{\rho}(z)\right|_{z=1}:=\lim _{z \rightarrow 1^{-}} \partial_{z} \hat{\rho}(z)<+\infty$ if and only if $\sum_{v} v \rho(v)<+\infty$. By taking the derivative with respect to $z$ on both sides of (4), and evaluating the resulting equation in $z=1$, one obtains

$$
\frac{d}{d t} M_{1}\left(f_{t}\right)=\alpha_{1} M_{1}\left(f_{t}\right)
$$

which gives (6). Analogous computations can be done to evaluate higher-order cumulant functions of the density $f_{t}$. An explicit expression of the variance of $f_{t}$ is presented in Proposition 6 .

Note that the condition

$$
\mathbb{E}[X+Y]=1
$$

implies that the mean remains constant in time, i.e. $M_{1}\left(f_{t}\right)=m_{0}$ for every $t>0$, as in Case 1 .

2.2. The quasi-invariant collision limit. The bilinear Boltzmann-like equation (2), fruitfully written in the form (4), is the starting point to obtain, in a well-established asymptotic procedure, simpler models that are reminiscent of the binary collision rules, and, while maintaining most of the properties of the nonlinear kinetic model, result to be linear. This kind of asymptotic procedure is close to the so-called grazing collision limit for the Boltzmann equation [48], and has been widely used in kinetic theory to obtain Fokker-Planck type equations to describe, among others, the cooling in granular gases [28,42], wealth distribution in a multi-agent society [21], price formation [16, 17], and opinion formation [26, 46].

This asymptotics procedure is based on the following assumptions. Given a small positive parameter $\epsilon$, assume that the random variables $X$ and $Y$ are defined by

$$
X=\eta_{1} \tilde{X}+\left(1-\eta_{1}\right) \quad \text { and } \quad Y=\eta_{2} \tilde{Y},
$$

where $\tilde{X}, \tilde{Y}, \eta_{1}, \eta_{2}$ are independent random variables, and, for some constants $b_{1}, b_{2}>0$ such that $b_{i} \epsilon \leq 1, i=1,2$, it holds

$$
P\left\{\eta_{i}=1\right\}=1-P\left\{\eta_{i}=0\right\}=b_{i} \epsilon, \quad i=1,2 .
$$

Note that by construction the laws of $X$ and $Y$ depend on $\epsilon$, so that $X=X_{\epsilon}$ and $Y=Y_{\epsilon}$. Nevertheless, for the shake of notational simplicity, the dependence in $\epsilon$ has been omitted. Since $\epsilon$ is assumed to be small, the post-collision quantities $\left(V_{1}^{\prime}, V_{2}^{\prime}\right)$ remain equal to the pre-collision ones $\left(V_{1}, V_{2}\right)$ with high probability. Indeed, with high probability, $X_{\epsilon}$ is equal to one and $Y_{\epsilon}$ to zero. In order to evaluate the mean value of $f_{t}(v)$ in correspondence to the collision defined by (8), observe that

$$
\mathbb{E}\left[X_{\epsilon}+Y_{\epsilon}\right]-1=\epsilon\left[b_{1}(\mathbb{E}[\tilde{X}]-1)+b_{2} \mathbb{E}[\tilde{Y}]\right]=: \epsilon \bar{\alpha}_{1} .
$$

Hence, (6) takes the form

$$
M_{1}\left(f_{t}\right)=\partial_{z} \hat{f}_{t}(1)=m_{0} e^{\bar{\alpha}_{1} \epsilon t} .
$$

This shows that, in presence of the small parameter $\epsilon$, to observe the same variation of the mean value corresponding to $\epsilon=1$, it is necessary to wait a longer time $\tau$, given by $\tau=t / \epsilon$.

By virtue of (8) one obtains

$$
\hat{p}_{X}(z)=z+\epsilon b_{1}\left(\hat{p}_{\tilde{X}}(z)-z\right) \text { and } \hat{p}_{Y}(z)=1+\epsilon b_{2}\left(\hat{p}_{\tilde{Y}}(z)-1\right) .
$$

Therefore, if $\hat{f}_{t}(z)=\hat{f}_{t, \epsilon}(z)$ is a solution of (4) corresponding to random variables $X$ and $Y$ defined in $(8)$, and the initial density $f_{0}$ has finite mean $m_{0}$, by expanding $\hat{f}_{t}\left(\hat{p}_{X}(z)\right)$ and $\hat{f}_{t}\left(\hat{p}_{Y}(z)\right)$ in Taylor's series around $z$ (around 1, respectively) gives

$$
\hat{f}_{t}\left(\hat{p}_{X}(z)\right)=\hat{f}_{t}(z)+\epsilon b_{1}\left(\hat{p}_{\tilde{X}}(z)-z\right) \partial_{z} \hat{f}_{t}(z)+\epsilon b_{1} R_{1, \epsilon}(t, z),
$$

and

$$
\hat{f}_{t}\left(\hat{p}_{Y}(z)\right)=\hat{f}_{t}(1)+\epsilon b_{2}\left(\hat{p}_{\tilde{Y}}(z)-1\right) \partial_{z} \hat{f}_{t}(1)+\epsilon b_{2} R_{2, \epsilon}(t, z),
$$

where $R_{1, \epsilon}$ and $R_{2, \epsilon}$ denote the remainders. Since by definition $\hat{f}_{t}(1)=1$, recalling also (9), the collision term in (4) takes the form

$$
\hat{f}_{t}\left(\hat{p}_{X}(z)\right) \hat{f}_{t}\left(\hat{p}_{Y}(z)\right)-\hat{f}_{t}(z)=\epsilon\left[b_{2} \hat{f}_{t}(z)\left(\hat{p}_{\tilde{Y}}(z)-1\right) M_{1}\left(f_{t}\right)+b_{1}\left(\hat{p}_{\tilde{X}}(z)-z\right) \partial_{z} \hat{f}_{t}(z)\right]+\epsilon R_{3, \epsilon}(t, z),
$$


where $R_{3, \epsilon}(t, z)$ denotes the new remainder term. In view of the previous remark on the evolution in time of the mean value, let us set $\tau=t / \epsilon$ and $\hat{g}_{\tau, \epsilon}(z)=\hat{f}_{\tau / \epsilon}(z)$. Then, $\hat{g}_{\tau, \epsilon}(z)$ satisfies the equation

$$
\partial_{\tau} \hat{g}_{\tau, \epsilon}(z)=\hat{g}_{\tau, \epsilon}(z) b_{2}\left(\hat{p}_{\tilde{Y}}(z)-1\right) M_{1}\left(g_{\tau, \epsilon}\right)+b_{1}\left(\hat{p}_{\tilde{X}}(z)-z\right) \partial_{z} \hat{g}_{\tau, \epsilon}(z)+R_{3, \epsilon}(\tau / \epsilon, z) .
$$

Let us assume that $R_{3, \epsilon} \rightarrow 0$ as $\epsilon \rightarrow 0$. Since $M_{1}\left(g_{\tau, \epsilon}\right)=m_{0} e^{\bar{\alpha}_{1} \tau}$, letting $\epsilon \rightarrow 0$, and defining $g_{\tau}(z)=\lim _{\epsilon \rightarrow 0} \hat{g}_{\tau, \epsilon}(z)$ shows that $g_{\tau}$ satisfies the equation

$$
\begin{aligned}
& \partial_{\tau} \hat{g}_{\tau}(z)=m_{0} e^{\bar{\alpha}_{1} \tau} b_{2}\left(\hat{p}_{\tilde{Y}}(z)-1\right) \hat{g}_{\tau}(z)+b_{1}\left(\hat{p}_{\tilde{X}}(z)-z\right) \partial_{z} \hat{g}_{\tau}(z), \\
& \hat{g}_{0}(z)=\hat{f}_{0}(z) .
\end{aligned}
$$

While the actual derivation of equation (11) is largely formal, every step can be made rigorous. We postpone a precise statement of the limit procedure and its detailed proof to Section 5 (cf. Proposition 10).

Here it is worth noticing that from the previous equation it follows that the mean of $g_{\tau}(\cdot)$ is $m_{0} e^{\bar{\alpha}_{1} \tau}$. Indeed recall that $y(\tau):=\left.\partial_{z} \hat{g}_{\tau}(z)\right|_{z=1}=M_{1}\left(g_{\tau}(\cdot)\right)$, so that deriving with respect to $z$ equation (11) and evaluating the resulting equation for $z=1$ one gets

$$
\dot{y}(\tau)=b_{1}(\mathbb{E}[\tilde{X}]-1) y(\tau)+b_{2} m_{0} e^{\bar{\alpha}_{1} \tau} \mathbb{E}[\tilde{Y}] .
$$

This implies

$$
y(\tau)=m_{0} e^{\bar{\alpha}_{1} \tau}+e^{b_{1} \mathbb{E}[\tilde{Y}] \tau}\left(y(0)-m_{0}\right),
$$

and the claim follows since the initial condition is $y(0)=m_{0}$.

2.3. The Lea-Coulson equation for mutation processes. Consider the special case in which $P\{\tilde{X}=2\}=1-P\{\tilde{X}=1\}=p$ and $P\{\tilde{Y}=1\}=1-P\{\tilde{Y}=0\}=q$. With this choice $p_{\tilde{X}}(z)-z=p z(z-1)$ and $p_{\tilde{Y}}(z)-1=q(z-1)$. By setting $\beta_{1}=p b_{1}+q b_{2}, \beta_{2}=b_{1} p$ and $\mu=b_{2} q m_{0}$, equation (11) takes the form

$$
\partial_{t} \hat{g}(t, z)=(z-1)\left\{\beta_{2} z \partial_{z} \hat{g}(t, z)+\mu e^{\beta_{1} t} \hat{g}(t, z)\right\} .
$$

Equation (12) is referred to in the literature as the Luria-Delbrück model. The model deals with the estimation of mutation rates, and has its origin in a series of classic experiments pioneered by Luria and Delbrück [39], which were at the basis of the construction of a mathematical model able to estimate them. The original model proposed by Luria and Delbrück assumed deterministic growth of mutant cells, which seemed a too stringent assumption to allow for efficient extraction of reliable information about mutation rates from experimental data. This shortcoming of the model of Luria and Delbrück was some year later remedied by a slightly different mathematical formulation proposed by Lea and Coulson [36], who adopted the Yule stochastic birth process to mimic the growth of mutant cells. In the ensuing decades the Lea-Coulson formulation occupied a so prominent place in the study of mutation rates that the Lea-Coulson formulation is now commonly referred to as the Luria-Delbrück model [50].

The classical linear procedure of growth of both normal and mutant cells, that give rise to equation (12) has been merged into the framework of kinetic theory in [33]. There, the mutation problem was described in terms of linear interactions, and the solution to the underlying linear Boltzmann equation was shown to converge towards the solution to the Lea-Coulson model. A precise statement of this convergence result has been subsequently done in [47].

While equation (12) coincides with the mathematical formulation of Lea and Coulson [50], it is important to remark that the meaning of the various (eventually time dependent) coefficients in (12) assume a different meaning depending on the original model from which this equation comes from.

In the original formulation, equation (12) is recovered through the following assumptions. The process starts at time $t=0$ with one normal cell and no mutants. Normal cells are assumed to grow deterministically at a constant rate $\beta_{1}$. Therefore the number of normal cells at time $t>0$ is $N(t)=e^{\beta_{1} t}$. Mutants grow deterministically at a constant rate $\beta_{2}$. If a mutant is generated by a normal cell at time $s>0$, then the clone spawned by this mutant will be of size $e^{\beta_{2}(t-s)}$ for any $t>s$. Mutations occur randomly at a rate proportional to $N(t)$. If $\mu$ denotes the per-cell 
per-unit-time mutation rate, then the standard assumption is that mutations occur in accordance with a Poisson process having the intensity function

$$
\nu(t)=\mu N(t)=\mu e^{\beta_{1} t} .
$$

Consequently, the expected number of mutations occurring in the time interval $[0, t)$ is

$$
\int_{0}^{t} \nu(s) d s=\frac{\mu}{\beta_{1}}\left(e^{\beta_{1} t}-1\right) .
$$

The formulation of Lea-Coulson differs from the previous Luria-Delbrück original formulation in that mutant cell growth is described by a stochastic birth process. If a mutant is generated by a normal cell at time $s>0$, then at any given time $t>s$ the size of the clone spawned by that mutant will have the same distribution as $Y(t-s)$, where $Y(s)$ is a Yule process having birth rate $\beta_{2}$ and satisfying $Y(0)=1$. In this way if $W(t)$ denotes the number of mutants existing at time $t$, then

$$
W(t)=\sum_{i=1}^{M(t)} Y_{i}\left(t-\tau_{i}\right), \quad \text { if } \quad M(t) \geq 1
$$

while

$$
W(t)=0 \quad \text { if } \quad M(t)=0 .
$$

Here, $\tau_{i}$ are the random times at which mutations occur, $M(t)$ stands for the mutation process which is a Poisson process with intensity function $\nu(t)$ given in $(13)$, and $\left(Y_{i}(s)_{i \geq 1}\right.$ is a sequence of independent Yule processes. In particular, the probability generating function of $W(t)$ satisfies (12) $[50]$.

Unlike the previous approach, the Lea-Coulson equation (12) follows here by linearizing a nonlinear equation of Boltzmann type based on classical binary interactions, and consequently from a nonlinear mechanism, in which the number of cells is subject only to duplication (the variable $X$ ) and mutation (the migration variable $Y$ ). In particular, the initial condition is here different, and the coefficients $\beta_{1}$ and $\beta_{2}$ are no more independent. In addition, the exponential in time term in front of the probability generating function $\hat{g}$ is in our derivation generated by the evolution of the mean value. Nevertheless, from a mathematical point of view, this derivation could share a new light on the large-time behaviour of the solution to (12), which is now connected with the solution of a nonlinear Boltzmann-type equation, that, unlike the linear model, possesses solutions of self-similar type. This new connection will be studied in more details in a companion paper.

2.4. Steady states. When $\mathbb{E}[X+Y]=1$, while $\mathbb{E}\left[X^{r}+Y^{r}\right]<+\infty$ for some $r>1$, one can prove that, for any given constant $m_{0}$, the kinetic model (2) possesses a steady state, namely a density satisfying

$$
f_{\infty}=Q^{+}\left(f_{\infty}, f_{\infty}\right)
$$

of mean $m_{0}$. Moreover, any solution $f_{t}$ with initial density $f_{0}$ of mean value $m_{0}$ converges (pointwise) to the corresponding stationary solution $f_{\infty}$ whenever $\sum_{v} v^{r} f_{0}(v)<+\infty$ (cf. Theorem 3 ). In Section 3.1 we shall show that $f_{t}$ converges to $f_{\infty}$ exponentially fast in time with respect to suitable Fourier metrics (cf. Theorem 2).

If $\mathbb{E}\left[X^{2}+Y^{2}\right]<+\infty$, one can recover an explicit formula for the second moment $M_{2}\left(f_{\infty}\right)$ of $f_{\infty}$. Indeed by combining Lemma 19 in Appendix with identity (15) one gets

$$
\begin{aligned}
M_{2}\left(f_{\infty}\right)= & M_{2}\left(Q^{+}\left(f_{\infty}, f_{\infty}\right)\right)=M_{2}\left(f_{\infty}\right)\left[\mathbb{E}[X]^{2}+\mathbb{E}[Y]^{2}\right] \\
& +2 \mathbb{E}[X] \mathbb{E}[Y] M_{1}\left(f_{\infty}\right)^{2}+[\operatorname{Var}(X)+\operatorname{Var}(Y)] M_{1}\left(f_{\infty}\right) .
\end{aligned}
$$

Since $M_{1}\left(f_{\infty}\right)=m_{0}$, it follows that

$$
\operatorname{Var}\left(f_{\infty}\right)=\frac{(\operatorname{Var}(X)+\operatorname{Var}(Y)) m_{0}}{1-\mathbb{E}[X]^{2}-\mathbb{E}[Y]^{2}} .
$$

While in principle one can recursively determine every integer moment of the steady state $f_{\infty}$, an explicit general expression for the p.g.f $\hat{f}_{\infty}$ is not available, with the exception of very simple 
situations. An explicit example is obtained by fixing $P\{X=1\}=1-P\{X=0\}=p$ and $P\{Y=1\}=1-P\{Y=0\}=q$ with $p+q=1$. In this case the steady state coincides with a Poisson distribution. To see this, recall that the p.g.f. of a Poisson distribution of mean $m_{0}$ is given by $\hat{f}_{\infty}(z)=\exp \left(m_{0}(z-1)\right)$. In the special case described above, $\hat{p}_{X}(z)=z p+1-p$ and $\hat{p}_{Y}(z)=z(1-p)+p$. Thus

$$
\hat{Q}^{+}\left(\hat{f}_{\infty}, \hat{f}_{\infty}\right)(z)=\exp \left(m_{0}\left(\hat{p}_{Y}(z)-1\right)\right) \exp \left(m_{0}\left(\hat{p}_{Y}(z)-1\right)\right)=\exp \left(m_{0}(z-1)\right)=\hat{f}_{\infty}(z) .
$$

One of the advantages of the quasi-invariant limit approximation is related to the possibility to obtain an explicit expression for the steady states. Indeed, if $b_{1}(\mathbb{E}[\tilde{X}]-1)+b_{2} \mathbb{E}[\tilde{Y}]=0$, the stationary equation reads

$$
m_{0} b_{2}\left(\hat{p}_{\tilde{Y}}(z)-1\right) \hat{g}_{\infty}(z)+b_{1}\left(\hat{p}_{\tilde{X}}(z)-z\right) \partial_{z} \hat{g}_{\infty}(z)=0 \quad z \in[0,1]
$$

and gives the explicit solution

$$
\hat{g}_{\infty}(z)=\exp \left\{-m_{0} \frac{b_{2}}{b_{1}} \int_{z}^{1} \frac{\left(1-\hat{p}_{\tilde{Y}}(s)\right)}{\left(\hat{p}_{\tilde{X}}(s)-s\right)} d s\right\} .
$$

In Proposition 11 we will present an interesting probabilistic interpretation of (16). Other aspects related to the steady states will be included there.

2.5. Steady states for horizontal gene transfer processes. Horizontal gene transfer (HGT) is believed to be the dominant component of genome innovation for bacteria. Recent estimates show that at least $32 \%$ of the genes in prokaryotes have been horizontally transferred [34, 35]. Today the study of the HGT can be tackled using a growing amount of genomic data. Nevertheless the study of mathematical models for HGT is still at the beginning. Among the few mathematical studies on the subject we mention the infinitely many genes model [7]. This model uses the coalescent theory in order to describe the underlying phylogenetic tree. A different approach has been considered in [31], which focuses on the description of a collisional mechanism which describes the time evolution of the gene-family abundance (the number of genes of a given family found in a genome). As already mentioned in the introduction, in the model a fixed (very large) number of species genomes interact pairwise randomly. When two species interact they can exchange genes by HGT by drawing them from each other with Bernoulli trials of probability $p_{h}$. In the same time, they draw from their own genome genes to be lost (with probability $p_{l}$ ) and duplicated (with probability $p_{d}$ ). In the model different families are considered subject to a stochastically independent evolution. Although this simplifying assumption is probably unrealistic, the effective rates $\left(p_{d}, p_{h}, p_{l}\right)$ can vary from family to family, giving rise to the observed diversity between families. An interesting result, which will be briefly described below, is that the model predicts an increased dispersion in family abundance as HGT becomes less relevant.

In [31] it is assumed that each gene can be transferred in a single copy, that corresponds to assume that $Y$ can take only the values $\{0,1\}$. In what follows we present a description of the steady states (in the quasi-invariant approximation) for a slightly more general situation, i.e. both $\tilde{X}$ and $\tilde{Y}$ take values $0,1,2$. For the sake of simplicity assume that $b_{1}=b_{2}=1$ and write

$$
P\{\tilde{X}=k\}=p_{k}, \quad P\{\tilde{Y}=k\}=q_{k} \quad k=0,1,2 .
$$

Since we need $\mathbb{E}[\tilde{X}+\tilde{Y}]=1$, we impose that

$$
p_{1}+q_{1}+2\left(p_{2}+q_{2}\right)=1 \text {. }
$$

With these choices it is immediate to conclude that

$$
\frac{\left(1-\hat{p}_{\tilde{Y}}(s)\right)}{\left(\hat{p}_{\tilde{X}}(s)-s\right)}=\frac{q_{1}+q_{2}+s q_{2}}{p_{0}-s p_{2}} .
$$

Recalling that (for $\beta \neq 0$ )

$$
\int_{z}^{1} \frac{a+b s}{\alpha+\beta s} d s=\frac{b}{\beta}(1-z)+\frac{a \beta-\alpha b}{\beta^{2}} \log \left(\frac{\alpha+\beta}{\alpha+z \beta}\right),
$$


when $p_{0}, p_{2}>0,(17)$ becomes

$$
\hat{g}_{\infty}(z)=e^{m_{0} \frac{q_{2}}{p_{2}}(z-1)}\left(\frac{1-p_{2} / p_{0}}{1-p_{2} / p_{0} z}\right)^{\frac{m_{0}}{p_{2}^{2}}\left(\left(q_{1}+q_{2}\right) p_{2}+p_{0} q_{2}\right)} .
$$

Note that $\mathbb{E}[\tilde{X}]<1$ implies both that $p_{0}>0$ and $p_{2}<p_{0}$, so that $1-p_{2} / p_{0}>0$. This proves that

$$
\hat{g}_{\infty}(y)=\hat{p}_{\Pi_{0}}(z) \hat{p}_{N}(z)
$$

where $p_{\Pi_{0}}(z)=e^{m_{0} \frac{q_{2}}{p_{2}}(z-1)}$ is the p.g.f. of a Poisson random variable $\Pi_{0}$ of mean $m_{0} q_{2} / p_{2}$ and $\hat{p}_{N}(z)$ is the p.g.f. of a Negative Binomial random variable of parameter $p=p_{2} / p_{0}$ and $r=\frac{m_{0}}{p_{2}^{2}}\left(\left(q_{1}+q_{2}\right) p_{2}+p_{0} q_{2}\right)$. Recall that a Negative binomial random variable of parameter $(p, r)$ has density

$$
p_{N}(k)=\left(\begin{array}{c}
k-r-1 \\
k
\end{array}\right)(1-p)^{r} p^{k} \quad k=0,1, \ldots
$$

In other words, in this case, the steady state $g_{\infty}$ is the density of the random variable

$$
V_{\infty}=\Pi_{0}+N
$$

where $\Pi_{0}$ and $N$ are independent. Since a negative binomial distribution can be represented as a compound Poisson distribution, i.e.

$$
N=\sum_{i=1}^{\Pi_{1}} L_{i},
$$

where $\Pi_{1}$ is a Poisson distribution of mean $r \log (1 /(1-p))$ and the $L_{i}$ 's are independent and identically distributed random variables, each one with logarithmic distribution

$$
p_{L_{i}}(k)=\frac{p^{k}}{k \log (1 /(1-p))} \quad(k=1,2, \ldots),
$$

one gets the representation

$$
V_{\infty}=\Pi_{0}+\sum_{i=1}^{\Pi_{1}} L_{i}
$$

If now we set $q_{2}=0$, we obtain

$$
\hat{g}_{\infty}(z)=\hat{p}_{N}(z)
$$

where $N$ is a Negative Binomial random variable of parameter $p=p_{2} / p_{0}$ and $r=m_{0} q_{1} / p_{2}$, i.e. of mean $m_{0}$ and variance $m_{0} p_{0} / q_{1}$. Note that this is the model introduced in [31] and briefly discussed above. In this case $p_{0}=p_{l}$ is the probability of loss of a gene, $p_{2}=p_{d}>0$ is the probability of duplication and $q_{1}=p_{h}$ is the probability of HGT. It is interesting to remark that in this case the resulting steady distribution depends only on the initial mean $m_{0}$ and on the ratio $p_{h} / p_{d}$. Moreover, the dispersion measured by the index Variance $/$ mean $=1+p_{d} / p_{h}$, increases as the probability $p_{h}$ of HGT decreases. The limit case is obtained when $p_{d}=p_{2}=0$.

Indeed, if $p_{2}=q_{2}=0$, direct computations show that $\hat{g}_{\infty}(y)=\exp \left\{m_{0}(z-1)\right\}$, namely that the steady states are given by Poisson distributions. In other words Poisson distributions are recovered in the case in which $p_{d}=0$, that is when no duplications occur.

Finally, although not relevant for the HGT model, let us consider the case in which $p_{2}=0$, while $q_{2}>0$. Here

$$
\hat{g}_{\infty}(y)=\exp \left\{\frac{m_{0}}{p_{0}}\left[\frac{q_{2}}{2} z^{2}+\left(q_{1}+q_{2}\right) z-\left(q_{1}+\frac{3}{2} q_{2}\right)\right]\right\}
$$

or, alternatively

$$
\hat{g}_{\infty}(y)=\exp \left\{\frac{2 m_{0}}{p_{0}\left(2 q_{1}+3 q_{2}\right)}\left[\hat{p}_{R}(z)-1\right]\right\}
$$

where

$$
\hat{p}_{R}(z)=q_{2}^{*} z^{2}+q_{1}^{*} z, \quad q_{2}^{*}:=q_{2} /\left(2 q_{1}+3 q_{2}\right), q_{1}^{*}=1-q_{2}^{*} .
$$


Since $\hat{p}_{R}(z)$ is the p.g.f. of a random variable $R$ taking values 1,2 with probability $q_{1}^{*}, q_{2}^{*}$, it follows that in this case the steady state $g_{\infty}$ is the density of the compound Poisson random variable

$$
V_{\infty}=\sum_{i=1}^{\Pi_{2}} R_{i},
$$

where $\Pi_{2}, R_{1}, R_{2}, \ldots$ are independent, $\Pi_{2}$ is distributed according to a Poisson distribution of mean $2 m_{0} /\left[p_{0}\left(2 q_{1}+3 q_{2}\right)\right]$ and the $R_{i}$ s have the same law of $R$.

\section{The kinetic equation: solutions, moments, steady states}

The remaining of the paper will be devoted to detailed proofs of various results about existence, uniqueness and asymptotic behavior of both the kinetic Boltzmann-type equation (4), and its quasi-invariant limit (11).

3.1. Existence and uniqueness of solutions. The unique solution of (4) can be written in a semi-explicit form by resorting to the so-called Wild series [49, 43].

Proposition 1. Let $f_{0}(v)$ be a probability density. Then, the initial value problem for equation (4), with initial condition $f_{0}$, has a unique global solution. For any given $t>0$, the unique solution $\hat{f}_{t}$ can be written as

$$
\hat{f}_{t}(z)=e^{-t} \sum_{n \geq 0}\left(1-e^{-t}\right)^{n} \hat{q}_{n}(z)
$$

where $\hat{q}_{0}(z)=\hat{f}_{0}(z)$, and, for any $n \geq 1$,

$$
\hat{q}_{n}(z):=\frac{1}{n} \sum_{j=0}^{n-1} \hat{q}_{j}\left(\hat{p}_{X}(z)\right) \hat{q}_{n-1-j}\left(\hat{p}_{Y}(z)\right) .
$$

Moreover, $\hat{f}_{t}$ remains a p.g.f. for any $t>0$.

Proof. The fact that $\hat{f}_{t}(z)$ is a solution can be checked directly. To prove uniqueness, let $\hat{f}_{t}$ and $\hat{g}_{t}$ be two solutions departing from the same initial value. Note that in place of (2) one can consider the same equation in integral form

$$
f_{t}(v)-f_{0}(v)=\int_{0}^{t}\left[Q^{+}\left(f_{s}, f_{s}\right)(v)-f_{s}(v)\right] d s \quad v \in \mathbb{N} .
$$

Define $u(t):=\sup _{z \in[0,1]}\left|\hat{f}_{t}(z)-\hat{g}_{t}(z)\right| \leq 2$. Using (18) one gets $u(t) \leq u(0)+2 \int_{0}^{t} u(s) d s$. Hence, Gronwall inequality implies $u(t) \leq u(0) e^{2 t}$. Since $u(0)=0$ it follows that $u(t)=0$. Finally, by induction one shows immediately that $\hat{q}_{n}$ is a p.g.f. for every $n \geq 0$. Hence $\hat{f}_{t}$ is a p.g.f..

An immediate consequence of the previous result is that the unique solution to equation (2) can be expressed as

$$
f_{t}(v)=e^{-t} \sum_{n \geq 0}\left(1-e^{-t}\right)^{n} q_{n}(v)
$$

where $q_{0}(v)=f_{0}(v)$ and, for any $n \geq 1$,

$$
q_{n}(v):=\frac{1}{n} \sum_{j=0}^{n-1} Q^{+}\left(q_{j}, q_{n-1-j}\right)(v) .
$$


3.2. Steady states and convergence to equilibrium. For any $r>0$, let us consider the following metric between probability distributions over non-negative integers

$$
d_{r}(f, g):=\sup _{z \in(0,1)} \frac{|\hat{f}(z)-\hat{g}(z)|}{|1-z|^{r}} .
$$

This is a natural adaptation of the well-known Fourier distance introduced in [29]. It is clear that convergence with respect to the metric $d_{r}$ yields convergence of p.g.f.'s and, hence, point-wise convergence of densities.

In dealing with general probability measures $f$ and $g$ on $[0,+\infty)$, it is also useful to introduce the weighted Laplace transform metric

$$
d_{r}^{*}(f, g):=\sup _{\xi>0} \frac{|\tilde{f}(\xi)-\tilde{g}(\xi)|}{|\xi|^{r}}
$$

where

$$
\tilde{f}(\xi)=\int e^{-\xi v} f(d v) \quad \text { and } \quad \tilde{g}(\xi)=\int e^{-\xi v} g(d v) .
$$

It is easy to see that $d_{r}$ and $d_{r}^{*}$ are (topologically) equivalent (cf. Lemma 18).

We remark that, in terms of the Laplace transform

$$
\tilde{f}_{t}(\xi)=\sum_{v} e^{-\xi v} f_{t}(v)
$$

the Boltzmann-type equation (2) takes the form

$$
\frac{\partial}{\partial t} \tilde{f}_{t}(\xi)=\tilde{f}_{t}\left(-k_{X}(\xi)\right) \tilde{f}_{t}\left(-k_{Y}(\xi)\right)-\tilde{f}_{t}(\xi), \quad \xi>0
$$

Now, $\tilde{f}_{0}(\xi)=\sum_{v} e^{-\xi v} f_{0}(v)$ is the Laplace transform of the initial density, and

$$
k_{X}(\xi)=\log \left(\mathbb{E}\left[e^{-\xi X}\right]\right) \quad \text { and } \quad k_{Y}(\xi)=\log \left(\mathbb{E}\left[e^{-\xi Y}\right]\right)
$$

are the cumulant functions of $X$ and $Y$.

Given a density $f$ on $\mathbb{N}$ (or more generally a probability measure $f$ on $\mathbb{R}^{+}$), $M_{r}(f)$ will denote the $r$-moment of $f$, expressed by $\sum_{v} v^{r} f(v)$ (or more generally by $\int v^{r} f(d v)$ ). To denote the variance of a random variable $Z$ we will write $\operatorname{Var}(Z)$. Moreover, if $f$ is a density (or a probability measure) $\operatorname{Var}(f)$ will denote the variance of a random variable $Z$ with law $f$, that is $\operatorname{Var}(f)=$ $M_{2}(f)-M_{1}(f)^{2}$. Finally, for $r \geq 1$, let us set

$$
\Delta_{r}:=\mathbb{E}[X]^{r}+\mathbb{E}[Y]^{r} \quad \text { and } \quad \alpha_{r}=\mathbb{E}[X]^{r}+\mathbb{E}[Y]^{r}-1 .
$$

Theorem 2 (Contraction in $d_{r}$ ). Assume that $\mathbb{E}\left[X^{r}+Y^{r}\right]<+\infty$ for some $r \in(1,2]$. Let $f_{t}^{(1)}$ and $f_{t}^{(2)}$ be two solutions of (2) with initial conditions $f_{0}^{(1)}$ and $f_{0}^{(2)}$ such that $M_{1}\left(f_{0}^{(1)}\right)=$ $M_{1}\left(f_{0}^{(2)}\right)=m_{0}$. Then for every $t>0$

$$
d_{r}\left(f_{t}^{(1)}, f_{t}^{(2)}\right) \leq d_{r}\left(f_{0}^{(1)}, f_{0}^{(2)}\right) e^{\alpha_{r} t} \quad \text { and } \quad d_{r}^{*}\left(f_{t}^{(1)}, f_{t}^{(2)}\right) \leq d_{r}^{*}\left(f_{0}^{(1)}, f_{0}^{(2)}\right) e^{\alpha_{r} t} .
$$

Proof. Assume that $d_{r}\left(f_{0}^{(1)}, f_{0}^{(2)}\right)<+\infty$, otherwise there is nothing to be proved. Recall that $\hat{p}_{X}(z)=\mathbb{E}\left[z^{X}\right]=\sum_{m \geq 0} z^{m} p_{X}(m)$ and $\hat{p}_{Y}(z)=\mathbb{E}\left[z^{Y}\right]=\sum_{m \geq 0} z^{m} p_{Y}(m)$. It is easy to check (cf. Thm. X1.1.1 in [27]) that

$$
1-\hat{p}_{X}(z)=(1-z) \sum_{m \geq 0} z^{m} \bar{P}_{X}(m)
$$

where $\bar{P}_{X}(m)=P(X>m)=1-\sum_{j=0}^{m} p_{j}$. The same result holds for $\hat{p}_{Y}$. This yields that, for every $z \in(0,1)$, one can write

$$
\frac{1-\hat{p}_{X}(z)}{1-z}=\sum_{m} z^{m} \bar{P}_{X}(m) \leq \sum_{m} \bar{P}_{X}(m)=\mathbb{E}[X]
$$


and $\left|1-\hat{p}_{Y}(z)\right| /(1-z) \leq \mathbb{E}[Y]$. Hence, for every $r>1$,

$$
\sup _{z \in(0,1)} \frac{\left|1-\hat{p}_{X}(z)\right|^{r}}{|1-z|^{r}}+\sup _{z \in(0,1)} \frac{\left|1-\hat{p}_{Y}(z)\right|^{r}}{|1-z|^{r}}=\mathbb{E}[X]^{r}+\mathbb{E}[Y]^{r}=\Delta_{r} .
$$

Now recall that, by Proposition 1 ,

$$
\hat{f}_{t}^{(i)}(z)=e^{-t} \sum_{n \geq 0}\left(1-e^{-t}\right)^{n} \hat{q}_{n}^{(i)}(z)
$$

where $\hat{q}_{0}^{(i)}(z)=\hat{f}_{0}^{(i)}(z)$ and, for any $n \geq 1$,

$$
\hat{q}_{n}^{(i)}(z):=\frac{1}{n} \sum_{j=0}^{n-1} \hat{q}_{j}^{(i)}\left(\hat{p}_{X}(z)\right) \hat{q}_{n-1-j}^{(i)}\left(\hat{p}_{Y}(z)\right) .
$$

Using the bound $\left|\hat{q}_{j}^{(i)}(z)\right| \leq 1$ for every $z \in(0,1)$ and $i=1,2$, we obtain

$$
\begin{aligned}
d_{r}\left(q_{n}^{(1)}, q_{n}^{(2)}\right) & \leq \frac{1}{n} \sum_{j=0}^{n-1} \sup _{z \in(0,1)} \frac{\left|\hat{q}_{j}^{(1)}\left(\hat{p}_{X}(z)\right) \hat{q}_{n-1-j}^{(1)}\left(\hat{p}_{Y}(z)\right)-\hat{q}_{j}^{(2)}\left(\hat{p}_{X}(z)\right) \hat{q}_{n-1-j}^{(2)}\left(\hat{p}_{Y}(z)\right)\right|}{|1-z|^{r}} \\
& \leq \frac{1}{n} \sum_{j=0}^{n-1} \sup _{z \in(0,1)} \frac{\mid q_{j}^{(1)}\left(\hat{p}_{X}(z)\right)-q_{j}^{(2)}\left(\hat{p}_{X}(z)\right) \frac{\left|1-\hat{p}_{X}(z)\right|^{r}}{|1-z|^{r}}}{\left|1-\hat{p}_{X}(z)\right|^{r}} \\
& +\frac{1}{n} \sum_{j=0}^{n-1} \sup _{z \in(0,1)} \frac{\left|q_{n-1-j}^{(1)}\left(\hat{p}_{Y}(z)\right)-q_{n-1-j}^{(2)}\left(\hat{p}_{Y}(z)\right)\right|}{\left|1-\hat{p}_{Y}(z)\right|^{r}} \frac{\left|1-\hat{p}_{Y}(z)\right|^{r}}{|1-z|^{r}} \\
& \leq \frac{\Delta_{r}}{n} \sum_{j=0}^{n-1} d_{r}\left(q_{j}^{(1)}, q_{j}^{(2)}\right) .
\end{aligned}
$$

Hence, if $d_{r}\left(f_{0}^{(1)}, f_{0}^{(2)}\right)=d_{r}\left(q_{0}^{(1)}, q_{0}^{(2)}\right)<+\infty$ then $d_{r}\left(q_{n}^{(1)}, q_{n}^{(2)}\right)<+\infty$ for every $n$ and by Lemma 14

$$
d_{r}\left(q_{n}^{(1)}, q_{n}^{(2)}\right) \leq d_{r}\left(f_{0}^{(1)}, f_{0}^{(1)}\right) \frac{\Gamma\left(\Delta_{r}+n\right)}{\Gamma\left(\Delta_{r}\right) \Gamma(n+1)} .
$$

Hence, using also (62),

$$
\begin{aligned}
d_{r}\left(f_{t}^{(1)}, f_{t}^{(1)}\right) & \leq \sum_{n \geq 0} e^{-t}\left(1-e^{-t}\right)^{n} d_{r}\left(q_{n}^{(1)}, q_{n}^{(2)}\right) \\
& \leq d_{r}\left(f_{0}^{(1)}, f_{0}^{(1)}\right) \sum_{n \geq 0} e^{-t}\left(1-e^{-t}\right)^{n} \frac{\Gamma\left(\Delta_{r}+n\right)}{\Gamma\left(\Delta_{r}\right) \Gamma(n+1)}=d_{r}\left(f_{0}^{(1)}, f_{0}^{(1)}\right) e^{\Delta_{r}-1} .
\end{aligned}
$$

To recover the second inequality in (23), recall that by (67), for every $r>1$,

$$
\Delta_{r}^{*}:=\sup _{\xi>0} \frac{\left|k_{X}(\xi)\right|^{r}}{|\xi|^{r}}+\sup _{\xi>0} \frac{\left|k_{Y}(\xi)\right|^{r}}{|\xi|^{r}}=\mathbb{E}[X]^{r}+\mathbb{E}[Y]^{r} .
$$

Using this identity, we can repeat the previous part of proof to conclude.

Theorem 3 (Convergence to steady states). Let $\mathbb{E}[X+Y]=1$, and $\mathbb{E}\left[X^{r}+Y^{r}\right]<+\infty$ for some $r \in(1,2]$. Then for every $m_{0}>0$, there exists a unique density $f_{\infty}$, with mean $m_{0}$ and finite moment of order $r$ satisfying $f_{\infty}=Q^{+}\left(f_{\infty}, f_{\infty}\right)$. Moreover, provided $M_{r}\left(f_{0}\right)<+\infty$, any solution $f_{t}$ departing from the initial density $f_{0}$ with mean $m_{0}$, converges in $d_{r}$-metric to the corresponding stationary solution $f_{\infty}$, and

$$
d_{r}\left(f_{t}, f_{\infty}\right) \leq d_{r}\left(f_{0}, f_{\infty}\right) e^{-\left|\alpha_{r}\right| t} .
$$

Proof. Let us observe that $\Delta_{r}<1$ for every $r>1$ whenever $\mathbb{E}[X+Y]=1$. Let $f_{0}=\delta_{m_{0}}$ and set $f_{n+1}=Q^{+}\left(f_{n}, f_{n}\right)$ for every $n \geq 0$. Since $\mathbb{E}[X+Y]=1$, one gets $M_{1}\left(f_{n}\right)=m_{0}$ for every $n$. Moreover, by Lemma 19 in the Appendix and the fact that $M_{1}\left(f_{n}\right)=m_{0}$ we have

$$
M_{r}\left(f_{n+1}\right) \leq A+\Delta_{r} M_{r}\left(f_{n}\right)
$$


where $A=m_{0}\left(\mathbb{E}\left[X^{r}\right]+\mathbb{E}\left[Y^{r}\right]\right)+m_{0}^{r}(2 \mathbb{E}[X] \mathbb{E}[Y])^{r / 2}$. Iterating on $n$ yields

$$
M_{r}\left(f_{n+1}\right) \leq A \sum_{i=0}^{n} \Delta_{r}^{i}+m_{0}^{r} \Delta^{n+1} \leq \frac{A}{1-\Delta_{r}}+m_{0}^{r} .
$$

Arguing as in (25) one gets

$$
d_{r}\left(f_{n+k}, f_{n}\right) \leq d_{r}\left(f_{n+k-1}, f_{n-1}\right) \Delta_{r},
$$

and hence

By Lemma 18 and (26)

$$
d_{r}\left(f_{n+k}, f_{n}\right) \leq d_{r}\left(f_{k}, f_{0}\right) \Delta_{r}^{n}
$$

$$
\sup _{k} d_{r}\left(f_{k}, f_{0}\right) \leq \sup _{k} \max \left\{2^{r+1}, B^{r} c_{r}\left(M_{r}\left(f_{k}\right)+M_{r}\left(f_{0}\right)\right)\right\} \leq C .
$$

Hence, the sequence $\left\{f_{n}\right\}_{n \geq 0}$ satisfies the Cauchy condition $d_{r}\left(f_{n}, f_{m}\right) \leq \epsilon$ for every $n, m \geq$ $N(\epsilon)$. Since $M_{1}\left(f_{n}\right)$ is bounded, by Prohorov theorem one obtains that $f_{n}$ is weakly compact (tight). Combining tightness with the Cauchy condition one concludes that there is a probability distribution $f_{\infty}$ such that $\hat{f}_{n} \rightarrow \hat{f}_{\infty}$ (pointwise). Since

$$
\hat{f}_{n+1}(z)=\hat{f}_{n}\left(\hat{p}_{X}(z)\right) \hat{f}_{n}\left(\hat{p}_{Y}(z)\right),
$$

taking $n \rightarrow+\infty$, one obtains $\hat{f}_{\infty}(z)=\hat{f}_{\infty}\left(\hat{p}_{X}(z)\right) \hat{f}_{\infty}\left(\hat{p}_{Y}(z)\right)$. The property $M_{r}\left(f_{\infty}\right)<+\infty$ follows from the fact that $\hat{f}_{n} \rightarrow \hat{f}_{\infty}$ and $\sup _{n} M_{r}\left(f_{n}\right)<+\infty$ (cf. Thm. 25.11 in [12]). Uniqueness of $f_{\infty}$ (in the class of distribution with finite $r$-moment) follows by Lemma 18 and Theorem 2.

3.3. Evolution of moments. We conclude this Section by giving explicit expressions for the evolution of the mean and the variance of the solution $f_{t}$.

Proposition 4 (Evolution of the mean). Let $f_{t}$ be the unique solution of equation (4) with initial condition $f_{0}$. If $m_{0}:=M_{1}\left(f_{0}\right)<+\infty$, then at any subsequent time $t>0$

$$
M_{1}\left(f_{t}\right)=m_{0} e^{\alpha_{1} t} .
$$

Proof. First of all note that

$$
\sum_{v} v Q^{+}\left(f_{0}, f_{0}\right)(v)=\mathbb{E}[X+Y] m_{0}=\Delta_{1} m_{0}
$$

Hence, using (20) one gets

$$
M_{1}\left(q_{n}\right)=\frac{\Delta_{1}}{n} \sum_{j=0}^{n-1} M_{1}\left(q_{j}\right) .
$$

By Lemma 14

$$
M_{1}\left(q_{n}\right)=m_{0} \frac{\Gamma\left(\Delta_{1}+n\right)}{\Gamma\left(\Delta_{1}\right) \Gamma(n+1)} .
$$

Thus, by (19) and (62),

$$
M_{1}\left(f_{t}\right)=e^{-t} \sum_{n \geq 0}\left(1-e^{-t}\right)^{n} M_{1}\left(q_{n}\right)=m_{0} e^{-t} \sum_{n \geq 0}\left(1-e^{-t}\right)^{n} \frac{\Gamma\left(\Delta_{1}+n\right)}{\Gamma\left(\Delta_{1}\right) \Gamma(n+1)}=e^{t\left(\Delta_{1}-1\right)} .
$$

Proposition 5. Let $\mathbb{E}\left[X^{r}+Y^{r}\right]<+\infty$ for some $r \in(1,2]$. If $f_{t}$ is the unique solution of equation (4) with initial condition $f_{0}$ such that $M_{1}\left(f_{0}\right)=m_{0}$ and $M_{r}\left(f_{0}\right)<+\infty$, then $t \mapsto M_{r}\left(f_{t}\right)$ is a continuous function and

$$
M_{r}\left(f_{t}\right) \leq M_{r}\left(f_{0}\right) e^{\alpha_{r} t}+e^{\alpha_{r} t} H(t) .
$$

Here $H(t)$ is given by

$$
H(t)=\int_{0}^{t}\left[\Delta_{r} m_{0} e^{\left(\alpha_{1}-\alpha_{r}\right) s}+(2 \mathbb{E}[X] \mathbb{E}[Y])^{r / 2} m_{0}^{r} e^{\left(r \alpha_{1}-\alpha_{r}\right) s}\right] d s .
$$


Proof. Let us start by proving that

$$
t \mapsto M_{r}\left(f_{t}\right)=e^{-t} \sum_{n \geq 0}\left(1-e^{-t}\right)^{n} M_{r}\left(q_{n}\right)
$$

is a continuous function on the set $\left\{t>0: M_{r}\left(f_{t}\right)<+\infty\right\}$, which turns out to be an open interval. Identity in equation (30) is immediate by (19) and Fubini theorem. Clearly, the series may diverge for some $t<+\infty$. We claim that there is $T=T\left(f_{0}, X, Y\right)>0$ such that $M_{r}\left(f_{t}\right) \leq+\infty$ for every $t \in[0, T]$. Using (20) and Lemma 19 one can check that

$$
M_{r}\left(q_{n}\right)=\frac{1}{n} \sum_{j=0}^{n-1} M_{r}\left(Q^{+}\left(q_{j}, q_{n-1-j}\right)\right)=\frac{\Delta_{r}}{n} \sum_{j=0}^{n-1} M_{r}\left(q_{j}\right)+\frac{B}{n} \sum_{j=0}^{n-1}\left[M_{1}\left(q_{j}\right)+M_{1}\left(q_{j}\right)^{r}\right],
$$

for a suitable $B=B(X, Y, r)<+\infty$. Now (29) and well known asymptotics for the Gamma function give

$$
M_{r}\left(q_{n}\right) \leq \frac{1}{n} \sum_{j=0}^{n-1} M_{r}\left(q_{j}\right)+B_{1} n^{\beta}
$$

for suitable $B_{1}$ and $\beta$. Since Lemma 15 yields that $M_{r}\left(q_{n}\right) \leq K^{n+1},(19)$ gives

$$
M_{r}\left(f_{t}\right)=e^{-t} \sum_{n \geq 0}\left(1-e^{-t}\right)^{n} M_{r}\left(q_{n}\right) \leq e^{-t} \sum_{n \geq 0}\left(1-e^{-t}\right)^{n} K^{n+1} .
$$

This shows that $I=\left\{t>0: M_{r}\left(f_{t}\right)<+\infty\right\}$ is non-empty. Let us show that $I$ is open. Note that $t \mapsto e^{t} M_{r}\left(f_{t}\right)$ is non-decreasing. Set $s_{0}:=\sup \left\{t: M_{r}\left(f_{t}\right)<+\infty\right\}$. If $s_{0}<+\infty$ and $s_{0} \in I$, one can consider the function $t \mapsto f_{s_{0}+t}$ as a solution of (2) with initial condition $f_{s_{0}}$ such that $M_{r}\left(f_{s_{0}}\right)<+\infty$. By the previous argument, it must be that $M_{r}\left(f_{t+\epsilon}\right)<+\infty$ for any $\epsilon \in\left[0, T\left(f_{s_{0}}, X, Y\right)\right]$, that gives a contradiction. The continuity on $I$ follows by observing that, for $z=\left(1-e^{-t}\right)$ with $t \in I, \sum_{n>0} z^{n} M_{r}\left(q_{n}\right)$ is a convergent power series.

Finally let us prove that, if $s_{0}:=\sup \left\{t: M_{r}\left(f_{t}\right)<+\infty\right\}<+\infty$ then $\lim _{t \rightarrow s_{0}^{-}} M_{r}\left(f_{t}\right)=+\infty$. If $s_{0}<+\infty$, the previous argument show that $s_{0} \notin I$, that is $\left(1-z_{0}\right) \sum_{n \geq 0} z_{0}^{n} M_{r}\left(q_{n}\right)=+\infty$ for $z_{0}=\left(1-e^{-s_{0}}\right)$. Then, Abel theorem yields that $\lim _{z \rightarrow z_{0}^{-}}(1-z) \sum_{n \geq 0} z^{n} M_{r}\left(q_{n}\right)=(1-$ $\left.z_{0}\right) \sum_{n \geq 0} z_{0}^{n} M_{r}\left(q_{n}\right)=+\infty$.

Now, by multiplying equation (2) by $e^{t}$ and integrating on $[0, t)$ we obtain

$$
e^{t} f_{t}(v)=f_{0}(v)+\int_{0}^{t} e^{s} Q^{+}\left(f_{s}, f_{s}\right)(v) d s .
$$

Hence, Fubini Theorem gives

$$
e^{t} M_{r}\left(f_{t}\right)=M_{r}\left(f_{0}\right)+\int_{0}^{t} e^{s} M_{r}\left(Q^{+}\left(f_{s}, f_{s}\right)\right) d s .
$$

Now note that, by Lemma 19 in the Appendix

$$
M_{r}\left(Q^{+}\left(f_{s}, f_{s}\right)\right) \leq \Delta_{r}\left(M_{1}\left(f_{s}\right)+M_{r}\left(f_{s}\right)\right)+B_{r} M_{1}\left(f_{s}\right)^{r},
$$

where $B_{r}=(2 \mathbb{E}[X] \mathbb{E}[Y])^{r / 2}$ and $\Delta_{r}=\mathbb{E}[X]^{r}+\mathbb{E}[Y]^{r}$. Since $M_{1}\left(f_{s}\right)=m_{0} e^{\alpha_{1} s}$, it follows

$$
M_{r}\left(Q^{+}\left(f_{s}, f_{s}\right)\right) \leq \Delta_{r} m_{0} e^{\alpha_{1} s}+B_{r} m_{0}^{r} e^{r \alpha_{1} s}+\Delta_{r} M_{r}\left(f_{s}\right) .
$$

Denote $m_{r}(t)=e^{t} M_{r}\left(f_{t}\right)$. We get

$$
m_{r}(t) \leq \Delta_{r} \int_{0}^{t} m_{r}(s) d s+m_{r}(0)+\int_{0}^{t} e^{s}\left[\Delta_{r} m_{0} e^{\alpha_{1} s}+B_{r} m_{0}^{r} e^{r \alpha_{1} s}\right] d s .
$$

Finally, thanks to Gronwall inequality which can be applied by virtue of the first part of the proof (see Lemma 21), it holds

$$
m_{r}(t) \leq m_{r}(0) e^{\Delta_{r} t}+\int_{0}^{t} e^{\Delta_{r}(t-s)} e^{s}\left[\Delta_{r} m_{0} e^{\alpha_{1} s}+B_{r} m_{0}^{r} e^{r \alpha_{1} s}\right] d s,
$$

and $s_{0}=+\infty$. 
Proposition 6 (Evolution of the variance). Let $\operatorname{Var}\left(f_{0}\right)<+\infty$ and assume that

$$
\mathbb{E}\left[X^{2}+Y^{2}\right]<+\infty \text {. }
$$

Then

$$
\begin{aligned}
\operatorname{Var}\left(f_{t}\right) & =M_{2}\left(f_{0}\right) e^{\alpha_{2} t}-m_{0}^{2} e^{2 \alpha_{1} t}+\beta m_{0}\left[\frac{e^{\alpha_{1} t}-e^{\alpha_{2} t}}{\alpha_{1}-\alpha_{2}} \mathbb{I}\left\{\alpha_{1} \neq \alpha_{2}\right\}+t e^{\alpha_{2} t} \mathbb{I}\left\{\alpha_{1}=\alpha_{2}\right\}\right] \\
& +2 \gamma m_{0}^{2}\left[\frac{e^{2 \alpha_{1} t}-e^{\alpha_{2} t}}{2 \alpha_{1}-\alpha_{2}} \mathbb{I}\left\{2 \alpha_{1} \neq \alpha_{2}\right\}+t e^{\alpha_{2} t} \mathbb{I}\left\{2 \alpha_{1}=\alpha_{2}\right\}\right]
\end{aligned}
$$

where

$$
\beta:=\operatorname{Var}(X)+\operatorname{Var}(Y) \quad \text { and } \quad \gamma:=\mathbb{E}[X] \mathbb{E}[Y] .
$$

Proof. Recall that, by Proposition 5, $t \mapsto M_{2}\left(f_{t}\right)$ is a continuous function. Using formula (69) in Lemma 19 we obtain

$$
M_{2}\left(Q^{+}\left(f_{s}, f_{s}\right)\right)=M_{2}\left(f_{s}\right)\left[\mathbb{E}[X]^{2}+\mathbb{E}[Y]^{2}\right]+M_{1}\left(f_{s}\right) \beta+2 \gamma M_{1}\left(f_{s}\right)^{2} .
$$

Combining (18), (27) and (33) one gets

$$
M_{2}\left(f_{t}\right)-M_{2}\left(f_{0}\right)=\int_{0}^{t}\left(\alpha_{2} M_{2}\left(f_{s}\right)+c(s)\right) d s,
$$

with

$$
c(s)=m_{0}\left(\beta e^{\alpha_{1} s}+2 \gamma m_{0} e^{2 \alpha_{1} s}\right) .
$$

Solving the equation one obtains

$$
M_{2}\left(f_{t}\right)=e^{\alpha_{2} t}\left(M_{2}\left(f_{0}\right)+\int_{0}^{t} c(s) e^{-\alpha_{2} s} d s\right),
$$

which implies

$$
\operatorname{Var}\left(f_{t}\right)=e^{\alpha_{2} t}\left(M_{2}\left(f_{0}\right)+\int_{0}^{t} c(s) e^{-\alpha_{2} s} d s\right)-m_{0}^{2} e^{2 \alpha_{1} t} .
$$

Simple computations then give (32).

Note that from $\mathbb{E}[X+Y]=1$ and $\mathbb{E}\left[X^{2}+Y^{2}\right]<+\infty$, it follows $\alpha_{2}<0=\alpha_{1}$ and $2 \gamma=-\alpha_{2}$. Then (32) implies

$$
\operatorname{Var}\left(f_{t}\right)=\frac{(\operatorname{Var}(X)+\operatorname{Var}(Y)) m_{0}}{\left|\alpha_{2}\right|}+\left[\operatorname{Var}\left(f_{0}\right)-\frac{(\operatorname{Var}(X)+\operatorname{Var}(Y)) m_{0}}{\left|\alpha_{2}\right|}\right] e^{-\left|\alpha_{2}\right| t},
$$

for every $t>0$.

\section{THE CASE OF INCREASING MEAN}

The asymptotic behavior of the solution to the Boltzmann equation (2) when the mean value is preserved in time has been fully described in Theorem 3. In this case, in fact, existence of a steady solution together with its main properties follows.

A completely different situation arises when $\alpha_{1}=\mathbb{E}[X+Y]-1>0$, as in Case 2. Now the mean value diverges to $+\infty$, and the usual way to extract information about the large-time behavior of the solution [42] is to scale the solution of equation (4), or equivalently (21), with respect to the mean, in such a way to maintain the mean constant. This allows to look for non-trivial asymptotics profiles of

where

$$
\tilde{h}(\xi, t)=\tilde{f}_{t}\left(\frac{\xi}{m(t)}\right)
$$

$$
m(t):=M_{1}\left(f_{t}\right)=m_{0} e^{\alpha_{1} t} .
$$

It is easy to verify that the Laplace transform $\tilde{h}$ of the scaled density satisfies the new equation

$$
\frac{\partial}{\partial t} \tilde{h}_{t}(\xi)+\tilde{h}_{t}(\xi)+\alpha_{1} \xi \frac{\partial}{\partial \xi} \tilde{h}_{t}(\xi)=\tilde{h}_{t}\left(-m(t) k_{X}\left(\frac{\xi}{m(t)}\right)\right) \tilde{h}_{t}\left(-m(t) k_{Y}\left(\frac{\xi}{m(t)}\right)\right) .
$$


Note that if $f^{(1)}$ and $f^{(2)}$ are two solutions of $(2)$, with initial conditions $f_{0}^{(1)}$ and $f_{0}^{(2)}$, while $h^{(1)}$ and $h^{(2)}$ are the respective scaled solutions, then

$$
d_{r}^{*}\left(h_{t}^{(1)}, h_{t}^{(2)}\right) \leq d_{r}^{*}\left(f_{t}^{(1)}, f_{t}^{(2)}\right) m(t)^{-r}=d_{r}^{*}\left(f_{t}^{(1)}, f_{t}^{(2)}\right) m_{0}^{-r} e^{-t r \alpha_{1}},
$$

and (23) yields that

$$
d_{r}^{*}\left(h_{t}^{(1)}, h_{t}^{(2)}\right) \leq m_{0}^{-r} d_{r}^{*}\left(h_{0}^{(1)}, h_{0}^{(2)}\right) e^{t\left(\alpha_{r}-r \alpha_{1}\right)} .
$$

In view of the previous bound, it appears natural to assume that

$$
\left(\alpha_{r} / r-\alpha_{1}\right)<0 \text { for some } r>1 .
$$

Condition (37) is reminiscent of the analogous condition for the existence of self-similar solutions of Kac like kinetic models, see $[2,14,42]$. Indeed, as we shall see, there is a precise connection between the asymptotic profile of the scaled solution of (2) and the asymptotic profile of the solution of a particular Kac like equation. It is important to remark that, since $\alpha_{r}=\mathbb{E}[X]^{r}+\mathbb{E}[Y]^{r}-1$, condition (37) is equivalent to ask that $r \mapsto \alpha_{r} / r$ is decreasing in $r=1$, i.e. $\left(\alpha_{r} / r\right)^{\prime}<0$ for $r=1$. Computing $\left(\alpha_{r} / r\right)^{\prime}$ one obtains that (37) is equivalent to

$$
\mathbb{E}[X](\log (\mathbb{E}[X])-1)+\mathbb{E}[Y](\log (\mathbb{E}[Y])-1)-1<0 .
$$

Clearly the previous relation is true if $\mathbb{E}[Y]<1$ and $\mathbb{E}[X]<1$, while is clearly false if one of the two expectations is bigger than $e$. In Figure 1 a numerical evaluation of the region where (37) is verified and $\mathbb{E}[X]+\mathbb{E}[Y]>1$ is reported. Additional information on the function $r \mapsto \alpha_{r} / r-\alpha_{1}$ can be found in Lemma 3.10 in [42].

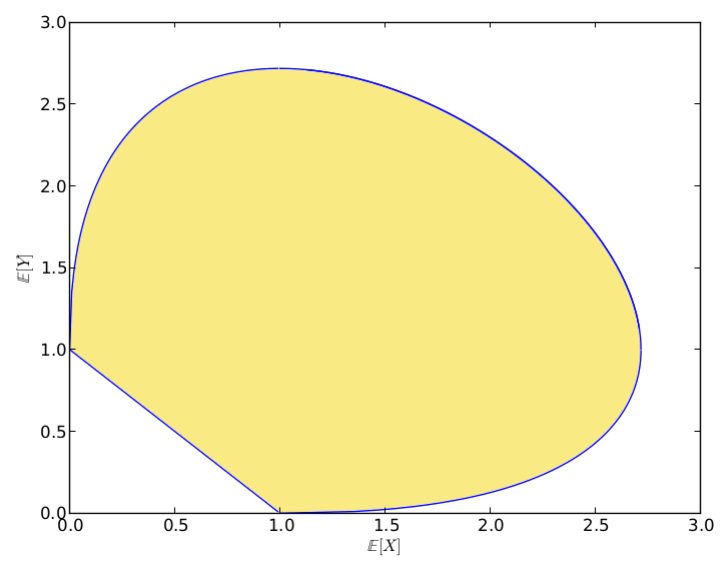

FiguRE 1. The interior of the curve (colored) represents the region in the plane $(\mathbb{E}[X], \mathbb{E}[Y])$ where $(37)$ is verified and $\mathbb{E}[X]+\mathbb{E}[Y]>1$.

Recalling that here $m(t) \rightarrow+\infty$, using (66), one can write

$$
-m(t) k_{X}(\xi / m(t))=\xi \mathbb{E}[X]+O\left(\xi^{r} m(t)^{1-r}\right),
$$

and hence,

$$
\lim _{t \rightarrow+\infty}\left(-m(t) k_{X}(\xi / m(t))=\xi \mathbb{E}[X] .\right.
$$

The same result holds for $Y$. In this way one can see that (at least formally) $\tilde{h}_{t}$ converges to the solution $\tilde{h}_{\infty}(\xi)=\int_{[0,+\infty)} e^{-\xi v} h_{\infty}(d v)$ of

$$
\tilde{h}_{\infty}(\xi)+\alpha_{1} \xi \frac{\partial}{\partial \xi} \tilde{h}_{\infty}(\xi)=\tilde{h}_{\infty}(\xi \mathbb{E}[X]) \tilde{h}_{\infty}(\xi \mathbb{E}[Y]) .
$$


For a precise statement see next Proposition 9. Now following [2, 6, 14], (39) is equivalent to the integral equation

$$
\tilde{h}_{\infty}(\xi)=\int_{0}^{1} \tilde{h}_{\infty}\left(\xi \mathbb{E}[X] \tau^{\alpha_{1}}\right) \tilde{h}_{\infty}\left(\xi \mathbb{E}[Y] \tau^{\alpha_{1}}\right) d \tau
$$

This shows that $h_{\infty}$ is the fixed point of the particular smoothing transformation:

$$
T\left(h_{\infty}, h_{\infty}\right)=\mathbf{L a w}\left(\mathbb{E}[X] U^{\alpha_{1}} V^{\prime}+\mathbb{E}[Y] U^{\alpha_{1}} V^{\prime \prime}\right)
$$

where $U, V^{\prime}, V^{\prime}$ are independent, $U$ is uniformly distributed on $(0,1)$, and $V^{\prime}, V^{\prime \prime}$ are distributed with law $h_{\infty}$. Note that

$$
\mathbb{E}\left[\mathbb{E}[X] U^{\alpha_{1}}+\mathbb{E}[Y] U^{\alpha_{1}}\right]=\frac{\mathbb{E}[X]+\mathbb{E}[Y]}{1+\alpha_{1}}=1
$$

Positive fixed points of smoothing transformations are deeply studied, mainly in connection with the so-called Branching Random Walk, see e.g. [1, 37]. It is worth noticing that in general - with the exception of few cases, see $[5,6]$ - there is no analytical expression of the law the of the fixed point of a given smoothing transformation. Even in our case it does not seem easy to obtain an explicit expression of $h_{\infty}$. An exception is the very special case when $E[X]=E[Y]=1$, where it is immediate to see that the solution is given by the exponential distribution

$$
\hat{h}_{\infty}(\xi)=\frac{1}{1-\xi \theta} .
$$

Nevertheless, it is always possible to recursively determine the exact expression of the integer moments of $h_{\infty}$. Indeed, using the fact that $h_{\infty}=T\left(h_{\infty}, h_{\infty}\right)$ and the Newton binomial formula, if $m_{i}:=\int v^{i} h_{\infty}(d v)<+\infty$ and $m_{1}=\int v h_{\infty}(d v)=1$, one gets

$$
m_{i}=\frac{1}{(\mathbb{E}[X]+\mathbb{E}[Y]-1) i+1-\mathbb{E}[X]^{i}-\mathbb{E}[Y]^{i}} \sum_{j=1}^{i-1}\left(\begin{array}{l}
i \\
j
\end{array}\right) \mathbb{E}[X]^{i} \mathbb{E}[Y]^{i-j} m_{j} m_{i-j}
$$

As we shall see in Proposition 7 below, $\int v^{r} h_{\infty}(d v)<+\infty$ if and only if $\alpha_{r} / r<\alpha_{1}$. Hence, $m_{i}<+\infty$ if and only if $(\mathbb{E}[X]+\mathbb{E}[Y]-1) i+1-\mathbb{E}[X]^{i}-\mathbb{E}[Y]^{i}>0$ so that (41) is well-defined. Note that when at least one of the two moments $\mathbb{E}[X]$ and $\mathbb{E}[Y]$ is strictly bigger than 1 , then there is $\bar{r}<+\infty$ such that $(\mathbb{E}[X]+\mathbb{E}[Y]-1) \bar{r}+1-\mathbb{E}[X]^{\bar{r}}-\mathbb{E}[Y]^{\bar{r}}<0$ and hence $\int v^{\bar{r}} h_{\infty}(d v)=+\infty$. This means that in this case $h_{\infty}$ has heavy tails. On the contrary if both $\mathbb{E}[X]$ and $\mathbb{E}[Y]$ are smaller than 1 , then $h_{\infty}$ has moments of all orders.

The fact that the steady state of (36) satisfies (40) suggests that, for a better understanding of the asymptotic behavior of equation (36), one can first to consider the large-time behavior of the equation

$$
\frac{\partial}{\partial t} \tilde{h}_{t}(\xi)+\tilde{h}_{t}(\xi)+\alpha_{1} \xi \frac{\partial}{\partial \xi} \tilde{h}_{t}(\xi)=\tilde{h}_{t}(\mathbb{E}[X] \xi) \tilde{h}_{t}(\mathbb{E}[Y] \xi)
$$

which is satisfied by the scaled solution $\tilde{h}(\xi, t)=\tilde{f}_{t}\left(e^{-\alpha_{1} t} \xi / m_{0}\right)$ of the equation

$$
\frac{\partial}{\partial t} \tilde{f}_{t}(\xi)+\tilde{f}_{t}(\xi)=\tilde{f}_{t}(\mathbb{E}[X] \xi) \tilde{f}_{t}(\mathbb{E}[Y] \xi)
$$

Equation (43) is associated to the very simple linear collision rule

$$
V_{i}^{\prime}=V_{i} \mathbb{E}[X]+V_{j} \mathbb{E}[Y], \quad V_{j}^{\prime}=V_{j} \mathbb{E}[X]+V_{i} \mathbb{E}[Y]
$$

and it is a special case of a general class of kinetic equations which have been deeply investigated, see for instance in $[2,3,14,40,44]$. It is immediate to realize that the interactions (44) correspond to a mean version of the interactions (1), and can be formally obtained from (1) by substituting $X$ and $Y$ with their mean values. Of course, this is consistent only if the mean values of $X$ and $Y$ are integer numbers. For this equation, one can resort to the results of [2] to obtain 
Proposition $7([2])$. Let $\mathbb{E}[X+Y]>1$, and let (37) hold true for some $r>1$. Then, there exists a unique solution to (40) such that $\int_{[0,+\infty)} v h_{\infty}(d v)=1$. Moreover, this solution satisfies $\int_{[0,+\infty)} v^{q} h_{\infty}(d v)<+\infty$ for $q>1$ if and only if $\alpha_{q} / q<\alpha_{1}$. Finally, if $\int v f_{0}(d v)=m_{0}<+\infty$ and $\tilde{f}_{t}$ is a solution of (43), then $\int v f_{t}(d v)=m_{0} e^{\alpha_{1} t}$ and $\tilde{f}_{t}\left(e^{-\alpha_{1} t} \xi / m_{0}\right)$ converges to $\tilde{h}_{\infty}(\xi)$ for every $\xi>0$ as $t \rightarrow \infty$.

Proof. The result follows from Proposition 2.1 and Theorem 2.2 in [2]. We have only to note that the spectral function $\mu(\cdot)$ defined in Section 2.2 of [2] in this case coincides with $r \mapsto \alpha_{r} / r-\alpha_{1}$.

One can adapt some results in $[2,40]$ to obtain

Proposition 8. Let $\mathbb{E}[X+Y]>1$ and assume that (37) holds true for some $r \in(1,2)$. Let $\tilde{f}_{t}$ be a solution of (43) with initial condition $f_{0}$ with finite $r$ moment. Then

$$
M_{r}\left(f_{t}\right) \leq C_{1} e^{\alpha_{r} t} .
$$

Moreover, if $\tilde{h}_{t}(\xi)=\tilde{f}_{t}\left(e^{-\alpha_{1} t} \xi / m_{0}\right), m_{0}=\int v f_{0}(d v)$, and $\tilde{h}_{\infty}(\xi)$ is the same as in Proposition 7 , then

$$
d_{r}^{*}\left(h_{t}, h_{\infty}\right) \leq C_{2} e^{\left(\alpha_{r}-r \alpha_{1}\right) t},
$$

for a suitable constant $C_{2}=C_{2}\left(f_{0}, h_{\infty}\right)$.

Proof. The first part of the thesis is a minor modification of Theorem 3.2 in [40], observing that in this case the function $\mathfrak{S}(r)$-defined in equation (32) of [40] - coincides with $\mathbb{E}[X]^{r}+\mathbb{E}[Y]^{r}-1=\alpha_{r}$. The last part of the thesis follows by a simple modification of Thm. 2.2 in [40]. See also Proposition 3.12 in [4]

In the next proposition we show that, under suitable assumptions, the scaled solutions of (2) and (43) merge when $t \rightarrow+\infty$. Combining this result with the known asymptotics for the scaled solution of (43) one obtains the asymptotic behavior of the scaled solution of (2) in Case 2.

Proposition 9 (Scaled solution). Let $\mathbb{E}[X+Y]>1$ and assume that (37) holds true for some $r \in(1,2)$. Let $\tilde{f}^{(1)}$ be a solution of $(21)$ with initial conditions $\tilde{f}_{0}$ with finite $r$-th moment and $\tilde{f}^{(2)}$ be a solution of (43) with the same initial condition $\tilde{f}_{0}$ with mean $m_{0}$. Set $\tilde{h}_{t}^{(i)}(\xi)=$ $\tilde{f}_{t}^{(i)}\left(e^{-\alpha_{1} t} \xi / m_{0}\right)$ for $i=1,2$. Then,

$$
d_{r}^{*}\left(h_{t}^{(1)}, h_{t}^{(2)}\right) \leq C\left(f_{0}\right)\left[t e^{\left(\alpha_{r}-r \alpha_{1}\right) t}+e^{-\alpha_{1} r t}\right] .
$$

In particular, $h_{t}^{(1)}$ converges weakly to the probability distribution $h_{\infty}$ given in Proposition 7.

Proof. We have

$$
\begin{aligned}
\xi^{-r} \mid & \tilde{f}_{t}^{(1)}\left(-k_{X}(\xi)\right) \tilde{f}_{t}^{(1)}\left(-k_{Y}(\xi)\right)-\tilde{f}_{t}^{(2)}(-\mathbb{E}[X] \xi) \tilde{f}_{t}^{(2)}(-\mathbb{E}[Y] \xi) \mid \\
& \leq \xi^{-r}\left|\tilde{f}_{t}^{(1)}\left(-k_{X}(\xi)\right) \tilde{f}_{t}^{(1)}\left(-k_{Y}(\xi)\right)-\tilde{f}_{t}^{(2)}\left(-k_{X}(\xi)\right) \tilde{f}_{t}^{(2)}\left(-k_{Y}(\xi)\right)\right| \\
& +\xi^{-r}\left|\tilde{f}_{t}^{(2)}\left(-k_{X}(\xi)\right) \tilde{f}_{t}^{(2)}\left(-k_{Y}(\xi)\right)-\tilde{f}_{t}^{(2)}\left(-k_{X}(\xi)\right) \tilde{f}_{t}^{(2)}(\mathbb{E}[Y] \xi)\right| \\
& +\xi^{-r}\left|\tilde{f}_{t}^{(2)}\left(-k_{X}(\xi)\right) \tilde{f}_{t}^{(2)}(\mathbb{E}[Y] \xi)-\tilde{f}_{t}^{(2)}(\mathbb{E}[X] \xi) \tilde{f}_{t}^{(2)}(\mathbb{E}[Y] \xi)\right| \\
& =: A_{1}+A_{2}+A_{3} .
\end{aligned}
$$

Now, arguing as in the proof of Theorem 2 ,

$$
A_{1} \leq \Delta_{r} d_{r}^{*}\left(f_{t}^{(1)}, f_{t}^{(2)}\right) .
$$

Moreover, using (68)

$$
A_{2} \leq \xi^{-r}\left|\tilde{f}_{t}^{(2)}\left(-k_{Y}(\xi)\right)-\tilde{f}_{t}^{(2)}(\mathbb{E}[Y] \xi)\right| \leq C(Y)\left[1+M_{r}\left(f_{t}^{(2)}\right)\right]
$$

and analogously

$$
A_{3} \leq C(X)\left[1+M_{r}\left(f_{t}^{(2)}\right)\right]
$$


At this stage recall that, by Proposition 8,

$$
M_{r}\left(f_{t}^{(2)}\right) \leq C_{1} e^{\alpha_{r} t} .
$$

Putting all estimates together one gets

$$
\begin{gathered}
H_{t}(\xi):=\xi^{-r}\left|\tilde{f}_{t}^{(1)}\left(-k_{X}(\xi)\right) \tilde{f}_{t}^{(1)}\left(-k_{Y}(\xi)\right)-\tilde{f}_{t}^{(2)}(-\mathbb{E}[X] \xi) \tilde{f}_{t}^{(2)}(-\mathbb{E}[Y] \xi)\right| \\
\leq \Delta_{r} d_{r}^{*}\left(f_{t}^{(1)}, f_{t}^{(2)}\right)+C_{3}\left[1+e^{\alpha_{r} t}\right] .
\end{gathered}
$$

Now, consider that

and

$$
\frac{\partial}{\partial t}\left(e^{t} \tilde{f}_{t}^{(1)}(\xi)\right)=e^{t} \tilde{f}_{t}^{(1)}\left(-k_{X}(\xi)\right) \tilde{f}_{t}^{(1)}\left(-k_{Y}(\xi)\right)
$$

$$
\frac{\partial}{\partial t}\left(e^{t} \tilde{f}_{t}^{(2)}(\xi)\right)=e^{t} \tilde{f}_{t}^{(2)}(\mathbb{E}[X] \xi) \tilde{f}_{t}^{(2)}(\mathbb{E}[Y] \xi) .
$$

Last, consider that $\tilde{f}_{0}^{(1)}=\tilde{f}_{0}^{(2)}$, and, using (46)

$$
e^{t} \xi^{-r}\left|\tilde{f}_{t}^{(1)}(\xi)-\tilde{f}_{t}^{(2)}(\xi)\right| \leq \int_{0}^{t} e^{s} H_{s}(\xi) d s \leq \int_{0}^{t} e^{s}\left[\Delta_{r} d_{r}^{*}\left(f_{s}^{(1)}, f_{s}^{(2)}\right)+C_{3}\left(1+e^{\alpha_{r} s}\right)\right] d s .
$$

Hence,

$$
\left.e^{t} d_{r}^{*}\left(f_{t}^{(1)}, f_{t}^{(2)}\right) \leq \Delta_{r} \int_{0}^{t} e^{s} d_{r}^{*}\left(f_{s}^{(1)}, f_{s}^{(2)}\right) d s+C_{3} \int_{0}^{t}\left[e^{s}+e^{\left(1+\alpha_{r}\right) s}\right)\right] d s .
$$

By Lemma 18,

$$
d_{r}^{*}\left(f_{t}^{(1)}, f_{t}^{(2)}\right) \leq c_{r}\left[M_{r}\left(f_{t}^{(1)}\right)+M_{r}\left(f_{t}^{(2)}\right)\right]
$$

and, by Propositions 5 and $8, t \mapsto M_{r}\left(f_{t}^{(1)}\right)+M_{r}\left(f_{t}^{(2)}\right)$ is a locally bounded function. Hence, Gronwall Lemma gives

$$
\left.d_{r}^{*}\left(f_{t}^{(1)}, f_{t}^{(2)}\right) \leq C_{3} e^{\left(\Delta_{r}-1\right) t} \int_{0}^{t}\left[e^{\left(1-\Delta_{r}\right) s}+1\right)\right] d s .
$$

If now $\alpha_{r} \neq 0$, it holds

$$
d_{r}^{*}\left(h_{t}^{(1)}, h_{t}^{(2)}\right) \leq C_{3} e^{-\alpha_{1} r t}\left[t e^{\alpha_{r} t}+\frac{1-e^{\alpha_{r} t}}{-\alpha_{r}}\right] \leq C_{4}\left[t e^{\left(-\alpha_{1} r+\alpha_{r}\right) t}+e^{-\alpha_{1} r t}\right] .
$$

In the remaining case $\alpha_{r}=0$ it holds $d_{r}^{*}\left(h_{t}^{(1)}, h_{t}^{(2)}\right) \leq C_{3} e^{-\alpha_{1} r t}$.

\section{The QUASI-INVARIANT LIMIT} 2.2 .

In this section we give precise statements and proofs of the limit procedure considered in Section

Proposition 10. Let $\hat{f}_{t, \epsilon}(z)$ be the solution of (4) for $X$ and $Y$ defined in (8) where $\mathbb{E}\left[\tilde{X}^{2}+\tilde{Y}^{2}\right]<$ $+\infty$ and $f_{0}$ has finite second moment and mean equal to $m_{0}$. Then, $\hat{g}(t, z)=\lim _{\epsilon \rightarrow 0} \hat{f}_{t / \epsilon, \epsilon}(z)$ satisfies

$$
\begin{aligned}
& \partial_{t} \hat{g}(t, z)=b_{1}\left(\hat{p}_{\tilde{X}}(z)-z\right) \partial_{z} \hat{g}(t, z)+b_{2} m_{0} e^{\bar{\alpha}_{1} t}\left(\hat{p}_{\tilde{Y}}(z)-1\right) \hat{g}(t, z), \\
& \hat{g}(0, z)=\hat{f}_{0}(z) .
\end{aligned}
$$

for $(t, z) \in(0,+\infty) \times[0,1]$, where $\bar{\alpha}_{1}=b_{1}(\mathbb{E}[\tilde{X}]-1)+b_{2} \mathbb{E}[\tilde{Y}]$. Moreover, the initial value problem for equation (47) has a unique solution, and

$$
M_{1}(g(t))=m_{0} e^{\bar{\alpha}_{1} t}
$$

Proof. Existence and uniqueness of a unique bounded solution in $Q=(0,1) \times(0, T)$, for every $T>0$ to equation (47) is known (cf. [25] for a detailed study of this type of linear equations).

Simple computations show that

$$
\alpha_{2}:=\mathbb{E}[X]^{2}+\mathbb{E}[Y]^{2}-1=2 \epsilon(\mathbb{E}[\tilde{X}]-1) b_{1}+\epsilon^{2}\left((\mathbb{E}[\tilde{X}-1])^{2} b_{1}^{2}+(\mathbb{E}[Y])^{2} b_{2}^{2}\right),
$$

while

$$
\beta:=\operatorname{Var}(X)+\operatorname{Var}(Y)=\epsilon\left(\mathbb{E}\left[(\tilde{X}-1)^{2}\right] b_{1}+\mathbb{E}\left[\tilde{Y}^{2}\right] b_{2}\right)-\epsilon^{2}\left((\mathbb{E}[\tilde{X}-1])^{2} b_{1}{ }^{2}+(\mathbb{E}[Y])^{2} b_{2}{ }^{2}\right),
$$


and

$$
\gamma:=\mathbb{E}[X] \mathbb{E}[Y]=\epsilon b_{2} \mathbb{E}[\tilde{Y}]\left(1+\epsilon b_{1}(\mathbb{E}[\tilde{X}]-1)\right) .
$$

Recall that $\alpha_{1}=\mathbb{E}[X]+\mathbb{E}[Y]-1=\epsilon \bar{\alpha}_{1}$. Hence, owing to (32), one finds that

$$
\operatorname{Var}\left(f_{t, \epsilon}\right) \leq G(\epsilon t)
$$

for a suitable function $G$ bounded on any fixed interval of time $[0, T]$. In addition we have

$$
\left|\partial_{z}^{2} \hat{f}_{t, \epsilon}(z)\right|=\left|\sum_{v \geq 2} v(v-1) z^{v-2} f_{t, \epsilon}(v)\right| \leq \sum_{v \geq 2} v^{2} f_{t, \epsilon}(v)=\operatorname{Var}\left(f_{t, \epsilon}\right)+M_{1}\left(f_{t, \epsilon}\right)^{2} .
$$

Recalling that that $M_{1}\left(f_{t, \epsilon}\right)=m_{0} e^{\bar{\alpha}_{1} \epsilon t}$ and using (48), for every $T<+\infty$ one gets the bound

$$
\sup _{t: t \epsilon \leq T}\left|\partial_{z}^{2} \hat{f}_{t, \epsilon}(z)\right| \leq C,
$$

where $C=C\left(f_{0}, \tilde{X}, \tilde{Y}, T\right)$ is independent of $\epsilon \leq \epsilon_{0}$ and $z$. Hence $\partial_{z} f_{t, \epsilon}(z)$ is locally uniformly (in $t$ and $\epsilon$ ) Lipshitz in $z$, which means that

$$
\left|\partial_{z} f_{t, \epsilon}(z)\right|_{z=z_{0}}-\left.\partial_{z} f_{t, \epsilon}(z)\right|_{z=z_{1}}|\leq L(T)| z_{0}-z_{1} \mid .
$$

for every $z_{0}, z_{1}$ in $[0,1]$ and every $t>0$ such that $t \epsilon<T$ and $\epsilon \leq \epsilon_{0}$.

To prove convergence, write

$$
\hat{f}_{t}\left(\hat{p}_{X}(z)\right)=\hat{f}_{t}(z)+\epsilon b_{1}\left(\hat{p}_{\tilde{X}}(z)-z\right) \partial_{z} \hat{f}_{t}(z)+\epsilon b_{1} R_{1, \epsilon}(t, z),
$$

and

$$
\hat{f}_{t}\left(\hat{p}_{Y}(z)\right)=\hat{f}_{t}(1)+\epsilon b_{2}\left(\hat{p}_{\tilde{Y}}(z)-1\right) \partial_{z} \hat{f}_{t}(1)+\epsilon b_{2} R_{2, \epsilon}(t, z) .
$$

In the previous equations,

$$
R_{1, \epsilon}(t, z)=\left(\hat{p}_{\tilde{X}}(z)-z\right)\left(\partial_{z} \hat{f}_{t}\left(z_{1, \epsilon, t}\right)-\partial_{z} \hat{f}_{t}(z)\right)
$$

for some $z_{1, \epsilon, t} \in[0,1]$ such that $\left|z-z_{1, \epsilon, t}\right| \leq \epsilon b_{1}\left|\hat{p}_{\tilde{X}}(z)-z\right|$ and

$$
R_{2, \epsilon}(t, z)=\left(\hat{p}_{\tilde{Y}}(z)-1\right)\left(\partial_{z} \hat{f}_{t}\left(z_{2, \epsilon, t}\right)-\partial_{z} \hat{f}_{t}(1)\right)
$$

for some $z_{2, \epsilon, t}$ such that $0 \leq 1+b_{2} \epsilon\left(\hat{p}_{\tilde{Y}}(z)-1\right) \leq z_{2, \epsilon, t} \leq 1$. Consequently

$$
\hat{f}_{t}\left(\hat{p}_{X}(z)\right) \hat{f}_{t}\left(\hat{p}_{Y}(z)\right)-\hat{f}_{t}(z)=\epsilon b_{2} \hat{f}_{t}(z)\left(\hat{p}_{\tilde{Y}}(z)-1\right) m(t)+\epsilon b_{1}\left(\hat{p}_{\tilde{X}}(z)-z\right) \partial_{z} \hat{f}_{t}(z)+\epsilon R_{3, \epsilon}(t, z) .
$$

Here, $m(t)=\partial_{z} \hat{f}_{t}(1)$ and

$$
\begin{aligned}
R_{3, \epsilon}(t, z) & =\epsilon b_{1} b_{2}\left(\hat{p}_{\tilde{X}}(z)-z\right)\left(\hat{p}_{\tilde{Y}}(z)-1\right) \partial_{z} \hat{f}_{t}(z) m(t)+\epsilon b_{1} b_{2}\left(\hat{p}_{\tilde{X}}(z)-z\right) \partial_{z} \hat{f}_{t}(z) R_{2, \epsilon}(t, z) \\
& +b_{2} R_{2, \epsilon}(t, z) \hat{f}_{t}(z)+R_{1, \epsilon}(t, z) b_{1}\left[1+\epsilon b_{2}\left(\hat{p}_{\tilde{Y}}(z)-1\right) m(t)+\epsilon b_{2} R_{2, \epsilon}(t, z)\right] .
\end{aligned}
$$

Let us set $\hat{g}_{t, \epsilon}(z)=\hat{f}_{t / \epsilon}(z)$. Then

$$
\partial_{t} \hat{g}_{t, \epsilon}(z)=\hat{g}_{t, \epsilon}(z) b_{2}\left(\hat{p}_{\tilde{Y}}(z)-1\right) M_{1}\left(g_{t, \epsilon}\right)+b_{1}\left(\hat{p}_{\tilde{X}}(z)-z\right) \partial_{z} \hat{g}_{t, \epsilon}(z)+R_{3, \epsilon}(t / \epsilon, z) .
$$

Using (49), for a suitable constant $C_{1}(T)$ we obtain

$$
\sup _{(z, t) \in Q} \sum_{i=1,2}\left|R_{i, \epsilon}(t / \epsilon, z)\right| \leq \epsilon C_{1}(T),
$$

so that

$$
\sup _{(z, t) \in Q}\left|R_{3, \epsilon}(t / \epsilon, z)\right| \leq \epsilon C_{2}(T) .
$$

Recalling now that for every $z \in[0,1]$ and every $t \in[0, T]$

$$
\partial_{z} \hat{g}_{t, \epsilon}(z) \leq M_{1}\left(g_{t, \epsilon}\right) \leq C_{3}(T),
$$

using this with (51) in equation (50) we obtain $\left|\partial_{t} \hat{g}_{t, \epsilon}(z)\right| \leq C_{4}(T)$. Summarizing, for a suitable bounded positive constant $C_{5}(T)$ we have

$$
\sup _{(z, t) \in[0,1] \times[0, T]}\left|\partial_{z} \hat{g}_{t, \epsilon}(z)\right|+\sup _{(z, t) \in[0,1] \times[0, T]}\left|\partial_{t} \hat{g}_{t, \epsilon}(z)\right| \leq C_{5}(T) .
$$


Combining Ascoli-Arzelá theorem with the Banach-Alaoglu-Bourbaki theorem one concludes that there is a subsequence $\hat{g}_{\cdot, \epsilon_{n}}(\cdot)$ converging strongly in $C^{0}([0,1] \times[0, T]$ ) (for every $T$ ) and weakly-* in $W^{1, \infty}(Q)$ to a function $\hat{g}$.

Since $R_{3, \epsilon} \rightarrow 0$ uniformly by (51), one can pass to the limit in (50) to obtain that $\hat{g}$ is a solution of (47). Next, uniqueness of the solution of (47) gives that the whole family $\hat{g}_{,, \epsilon}$ converges to $\hat{g}$ strongly in $C^{0}([0,1] \times[0, T])$, for every $T>0$. Finally, since the mean and variance of $g_{t, \epsilon}$ are uniformly bounded with respect to $\epsilon$, the sequence $\left\{g_{t, \epsilon}\right\}_{\epsilon \geq 0}$ is tight, and the limit function $g(\cdot, t)$ is also a p.g.f. with finite variance.

Define $\phi_{X}(z)=\sum_{k \geq 0} z^{k} P\{\tilde{X}>k\}$ and $\phi_{Y}(z)=\sum_{k \geq 0} z^{k} P\{\tilde{Y}>k\}$. Setting $m=\mathbb{E}[\tilde{X}]$, if $b_{1}(\mathbb{E}[\tilde{X}]-1)+b_{2} \mathbb{E}[\tilde{Y}]=0$, one can write $(1-m) b_{1} / b_{2}=\mathbb{E}[\tilde{Y}]$ so that $P^{*}(z):=m^{-1} \phi_{X}(z)$ and $Q^{*}(z):=b_{2} / b_{1}(1-m)^{-1} \phi_{Y}(z)$ are p.g.f.. It is worth noticing that, if $b_{1}=b_{2}=1, P^{*}$ and $Q^{*}$ are the p.g.f. of the so called renewal distribution of $p_{X}$ and $p_{Y}$. Recall also that given a probability density $g$ on $\mathbb{N}$ with finite mean $M_{1}(g)=\sum_{k} k g(k)$, its size-biased version is defined by

$$
g^{*}(k):=\frac{k g(k)}{M_{1}(g)} .
$$

Proposition 11. Under the same assumptions of Proposition 10, if $b_{1}(\mathbb{E}[\tilde{X}]-1)+b_{2} \mathbb{E}[\tilde{Y}]=0$, then the unique $C^{1}$-function $\hat{g}_{\infty}(z)$ with $\hat{g}_{\infty}(1)=1$ which is a stationary solution of $(47)$, i.e. the unique solution of (16), is given by

$$
\hat{g}_{\infty}(z)=\exp \left\{-m_{0} \frac{b_{2}}{b_{1}} \int_{z}^{1} \frac{\left(1-\hat{p}_{\tilde{Y}}(s)\right)}{\left(\hat{p}_{\tilde{X}}(s)-s\right)} d s\right\} .
$$

Moreover, $\hat{g}_{\infty}$ is the p.g.f. of an infinite divisible distribution $g_{\infty}$ over the non-negative integers. Finally, $g_{\infty}$ is the density of a random variable $V_{\infty}$ satisfying

$$
V_{\infty}^{*} \stackrel{d}{=} V_{\infty}+1+\zeta+\sum_{k=1}^{\gamma} \xi_{k}
$$

where $V_{\infty}^{*}$ is the size biased version of $V_{\infty}, \zeta$ has p.g.f. $Q^{*}, \xi_{k}$ has p.g.f. $P^{*}$ for every $k \geq 1$, $\gamma$ has geometric distribution of parameter $m=\mathbb{E}[\tilde{X}]$ and $V_{\infty}, \zeta, \gamma, \xi_{1}, \xi_{2}, \ldots$ are stochastically independent.

Proof. A stationary distribution $\hat{g}_{\infty}$ of (47) must satisfy (16). That is

$$
\partial_{z} \hat{g}_{\infty}(z)=\frac{m_{0} b_{2}\left(1-\hat{p}_{\tilde{Y}}(z)\right)}{b_{1}\left(\hat{p}_{\tilde{X}}(z)-z\right)} \hat{g}_{\infty}(z)
$$

that gives (52). Now note that, using (24) and the analogous relation for $Y$, one gets

$$
R(z):=\frac{b_{2}\left(1-\hat{p}_{\tilde{Y}}(z)\right)}{b_{1}\left(\hat{p}_{\tilde{X}}(z)-z\right)}=\frac{b_{2} \phi_{Y}(z)}{b_{1}\left(1-\phi_{X}(z)\right)}=\frac{(1-m) Q^{*}(z)}{1-m P^{*}(z)}=G_{m}\left(P^{*}(z)\right) Q^{*}(z)
$$

where

$$
G_{m}(s)=\frac{1-m}{1-m s}
$$

is the p.g.f. of a geometric random variable of parameter $m$. In other words $R(z)$ is the p.g.f. of the random variable

$$
\zeta+\sum_{k=1}^{\gamma} \xi_{k}
$$

We proved that

$$
\hat{g}_{\infty}(z)=\exp \left\{-m_{0} \int_{z}^{1} R(s) d s\right\},
$$

where $R$ is a p.g.f. and hence $m_{0} R$ is an absolutely monotone function. This show that $\hat{g}_{\infty}(z)$ is a p.g.f. of an infinite divisible distribution $g_{\infty}$ over the non-negative integers. Indeed a function $h$ with $h(0)>0$ is the p.g.f. of an infinitely divisible distribution over the on-negative integers if and only if $h(z)=\exp \left\{\int_{z}^{1} H(s) d s\right\}$ for an absolutely monotone function $H$ (cf. Thm 4.2 [45]). Finally, 
note that $m_{0}^{-1} \partial_{z} \hat{g}_{\infty}(z)=z^{-1} g_{\infty}^{*}(z)$ with $g_{\infty}^{*}(z)=\sum_{k \geq 0} z^{k} g_{\infty}^{*}(k)$ and $g_{\infty}^{*}(k)=k g_{\infty}(k) / m_{0}$, that is the size-biased version of $g_{\infty}$. At this stage one can re-write (16) as

$$
z R(z) \hat{g}_{\infty}(z)=g_{\infty}^{*}(z) .
$$

Therefore, in terms of random variables, (16) takes the form

$$
V_{\infty}^{*} \stackrel{d}{=} V_{\infty}+1+\zeta+\sum_{k=1}^{\gamma} \xi_{k}
$$

where $V_{\infty}$ is the size biased version of $V_{\infty}$.

Adapting Lemma 2.2 in [18] one gets a further result.

Lemma 12. Let $\left(\tilde{f}_{n}\right)_{n},\left(\tilde{g}_{n}\right)_{n}, \tilde{f}, \tilde{g}$ be the Laplace transform of probability measures on the positive real axis, such that $\tilde{f}_{n} \rightarrow \tilde{f}, \tilde{g}_{n} \rightarrow \tilde{g}, M_{1}\left(f_{n}\right)=M_{1}\left(g_{n}\right)=m_{0}$, and for some $\epsilon>0$

$$
\sup _{n}\left\{\max \left\{\int_{\mathbb{R}^{+}} v^{r+\epsilon} d f_{n}, \int_{\mathbb{R}^{+}} v^{r+\epsilon} d g_{n}\right\}\right\}=M_{\epsilon}<+\infty \text {. }
$$

Then

$$
d_{r}^{*}(f, g) \leq \liminf _{n} d_{r}^{*}\left(f_{n}, g_{n}\right) .
$$

Proof. Recall that pointwise convergence of Laplace transforms yields uniform convergence on every compact set, hence, for every $0<\delta<R<+\infty$,

$$
C_{n}=C_{n}(\delta, \epsilon):=\sup _{\xi \in[\delta, R]}\left|\tilde{f}_{n}(\xi)-\tilde{f}(\xi)\right|+\sup _{\xi \in[\delta, R]}\left|\tilde{g}_{n}(\xi)-\tilde{g}(\xi)\right| \rightarrow 0 .
$$

Moreover, since pointwise convergence of Laplace transform yields weak convergence of distribution (cf. Theorem 25.11 and Corollary 25.12 in [12]), using condition (55) one obtains $M_{1}(f)=M_{1}(g)=$ $m_{0}$ and

$$
\max \left\{\int v^{r+\epsilon} f(d v), \int v^{r+\epsilon} g(d v)\right\} \leq M_{\epsilon} .
$$

Using again (55), (64) yields

$$
\left|\tilde{f}_{n}(\xi)-\tilde{f}(\xi)\right| \xi^{-r} \leq\left|\tilde{f}_{n}(\xi)-1-m_{0} \xi\right| \xi^{-r}+\left|\tilde{f}(\xi)-1-m_{0} \xi\right| \xi^{-r} \leq 2 c_{r+\epsilon} \xi^{\epsilon} M_{\epsilon} .
$$

Hence

$$
\sup _{\xi \in(0, \delta)}\left|\tilde{f}_{n}(\xi)-f(\xi)\right| \xi^{-r} \leq 2 c_{r+\epsilon} \delta^{\epsilon} M_{\epsilon} .
$$

Now, consider that

$$
\begin{aligned}
d_{r}^{*}\left(f_{n}, f\right) & \leq \max \left\{\sup _{\xi \in(0, \delta)}\left|\tilde{f}_{n}(\xi)-f(\xi)\right| \xi^{-r}, \sup _{\xi \in[\delta, R)}\left|\tilde{f}_{n}(\xi)-f(\xi)\right| \xi^{-r}, \sup _{\xi \geq R}\left|\tilde{f}_{n}(\xi)-f(\xi)\right| \xi^{-r}\right\} \\
& \leq \max \left\{2 c_{r+\epsilon} \delta^{\epsilon} M_{\epsilon}, C_{n}, \frac{2}{R^{r}}\right\}
\end{aligned}
$$

Since the same estimate holds for $d_{r}^{*}\left(g_{n}, g\right)$,

$$
\lim _{n}\left[d_{r}^{*}\left(f_{n}, f\right)+d_{r}^{*}\left(g_{n}, g\right)\right]=0 .
$$

To conclude it suffices to observe that

$$
d_{r}^{*}(f, g) \leq d_{r}^{*}\left(f_{n}, g_{n}\right)+d_{r}^{*}\left(f_{n}, f\right)+d_{r}^{*}\left(g_{n}, g\right) .
$$

Theorem 13. Let $X$ and $Y$ be defined as in (8), with $b_{1}=b_{2}$, and $\tilde{X}$ and $\tilde{Y}$ satisfying (7)-(31). Let $f_{t, \epsilon}^{(i)}, i=1,2$, be two solutions of (2) with initial conditions $f_{0}^{(i)}$ with mean equal to $m_{0}$ and finite second moment. Let $g_{t}^{(1)}$ and $g_{t}^{(2)}$ be the corresponding quasi invariant collision limits of Proposition 10. Then, for every $1<r<2$,

$$
d_{r}^{*}\left(g_{t}^{(1)}, g_{t}^{(2)}\right) \leq d_{r}^{*}\left(g_{0}^{(1)}, g_{0}^{(2)}\right) e^{-r(1-\mathbb{E}[\tilde{X}])^{r-1} t} .
$$


Proof. We first o bserve that $\mathbb{E}[\tilde{X}]+\mathbb{E}[\tilde{Y}]=1$ i mplies $1-\mathbb{E}[\tilde{X}]>0$. Explicit computations and Taylor formula give

$$
\begin{aligned}
\alpha_{r} & =\mathbb{E}[X]^{r}+\mathbb{E}[Y]^{r}-1=[1+\epsilon(\mathbb{E}[\tilde{X}]-1)]^{r}+\epsilon^{r} \mathbb{E}[\tilde{X}]^{r}-1 \\
& =r \epsilon(\mathbb{E}[\tilde{X}]-1)^{r-1}+\frac{r(r-1)}{2} \epsilon^{2}(\mathbb{E}[\tilde{X}]-1)^{2}\left(1+Q_{\epsilon}\right)^{r-2}+\epsilon^{r} \mathbb{E}[\tilde{X}]^{r},
\end{aligned}
$$

with $\left|Q_{\epsilon}\right|<\epsilon(1-\mathbb{E}[\tilde{X}])$. Therefore

$$
a_{r}=r \epsilon(1-\mathbb{E}[\tilde{X}])^{r}+o(\epsilon)
$$

Combining (35), (5) and (59) it follows

$$
M_{2}\left(f_{t / \epsilon, \epsilon}^{(i)}\right) \leq C\left[1+e^{-\alpha_{2}(\epsilon) t / \epsilon}\right] \leq C^{\prime},
$$

for every $t>0$ and $\epsilon>0$. Since $\tilde{g}_{t}^{(i)}(z)=\lim _{\epsilon \rightarrow 0} \tilde{f}_{t / \epsilon, \epsilon}^{(i)}(z)$, combining Theorem 2, (56) and (59)

$$
d_{r}^{*}\left(g_{t}^{(1)}, g_{t}^{(2)}\right) \leq \liminf _{\epsilon} d_{r}^{*}\left(f_{t / \epsilon}^{(1)}, f_{t / \epsilon}^{(2)}\right) \leq d_{r}^{*}\left(g_{0}^{(1)}, g_{0}^{(2)}\right) e^{-r(1-\mathbb{E}[\tilde{X}])^{r-1} t} .
$$




\section{Appendix A}

A.1. Recurrence relations. We state without proof two previously used results.

Lemma 14. Let $0<a_{0}<+\infty$, and, for every $n \geq 1$, let $a_{n} \geq 0$ satisfy, for some $0<\Delta<+\infty$, the inequality

$$
a_{n} \leq \frac{\Delta}{n} \sum_{j=0}^{n-1} a_{j} .
$$

Then, for every $n \geq 1$

$$
a_{n} \leq a_{0} \frac{\Gamma(\Delta+n)}{\Gamma(\Delta) \Gamma(n+1)} .
$$

Moreover the equality sign in (60) implies the equality sign in (61).

Lemma 15. Let $0<a_{0}<+\infty$, and, for every $n \geq 1$, let $a_{n} \geq 0$ satisfy the inequality

$$
a_{n} \leq \frac{A}{n} \sum_{j=0}^{n-1} a_{j}+B n^{\beta},
$$

for some $0<A, B, \beta<+\infty$. Then there is $K=K\left(A, B, \beta, a_{0}\right) \geq 1$ such that for every $n \geq 0$

$$
a_{n} \leq K^{n+1} \text {. }
$$

We conclude this short section by recalling that, for any $t>0$ and $\Delta>0[30]$

$$
e^{(\Delta-1) t}=\sum_{n \geq 0} e^{-t}\left(1-e^{-t}\right)^{n} \frac{\Gamma(\Delta+n)}{\Gamma(\Delta) \Gamma(n+1)} .
$$

A.2. Inequalities for Laplace transforms and cumulants. Given a random variable $Z$ taking values in $\mathbb{R}^{+}$let us set, for $\xi>0$

$$
L_{Z}(\xi)=\mathbb{E}\left[e^{-\xi Z}\right] \quad \text { and } \quad k_{Z}(\xi)=\log \left(L_{Z}(\xi)\right) .
$$

Since for all $x>0$

$$
\left|e^{-x}-1\right| \leq|x|,
$$

and for every $r \in(1,2)$ one can find a constant $c_{r}$ such that

$$
\left|e^{-x}-1+x\right| \leq c_{r}|x|^{r},
$$

whenever $\mathbb{E}\left[Z^{r}\right]<+\infty$ and $\xi>0$ it follows

$$
\left|L_{Z}(\xi)-1-\xi \mathbb{E}[Z]\right| \leq c_{r} \xi^{r} \mathbb{E}\left[Z^{r}\right]
$$

and, if $\mathbb{E}[Z]<+\infty$,

$$
\left|L_{Z}(\xi)-1\right| \leq \xi \mathbb{E}[Z]
$$

The following lemmas contain useful bounds for cumulants.

Lemma 16. Let $\mathbb{E}\left[Z^{r}\right]<+\infty$ for some $r \in(1,2)$. Then, if $0 \leq \xi \leq(2 \mathbb{E}[Z])^{-1}$,

$$
\left|k_{Z}(\xi)+\xi \mathbb{E}[Z]\right| \leq c_{r} \xi^{r} \mathbb{E}\left[Z^{r}\right]+\xi^{2}(\mathbb{E}[Z])^{2} .
$$

Moreover

$$
\sup _{\xi>0} \frac{\left|k_{Z}(\xi)\right|^{r}}{|\xi|^{r}}=\mathbb{E}[Z]^{r}
$$

Proof. For every complex number $z$ write

$$
\log (1+z)=z(1+R(z)) .
$$

Then $|R(z)| \leq|z|$ for every $z$ such that $|z| \leq 1 / 2$ (cf. Proposition 8.46 in [15]). Thanks to (65), $0 \leq \xi \leq(2 \mathbb{E}[Z])^{-1}$ implies

$$
\left|L_{Z}(\xi)-1\right| \leq|\xi| \mathbb{E}[Z] \leq \frac{1}{2}
$$


By virtue of (64) and (65)

$$
\begin{aligned}
\left|k_{Z}(\xi)+\xi \mathbb{E}[Z]\right| & =\left|\log \left(1+L_{Z}(\xi)-1\right)+\xi \mathbb{E}[Z]\right|=\left|\left(L_{Z}(\xi)-1\right)\left(1+R\left(L_{Z}(\xi)-1\right)\right)+\xi \mathbb{E}[Z]\right| \\
& \leq\left|L_{Z}(\xi)-1+\xi \mathbb{E}[Z]\right|+\left[L_{Z}(\xi)-1\right]^{2} \leq x_{r} \xi^{r} \mathbb{E}\left[Z^{r}\right]+\xi^{2}(\mathbb{E}[Z])^{2}
\end{aligned}
$$

To obtain (67), note that $\xi \mapsto L_{Z}(\xi)=\mathbb{E}\left[e^{-\xi Z}\right]$ is a non-increasing function and $\mathbb{E}\left[e^{-\xi Z}\right] \leq 1$. Hence $\xi \mapsto\left|\log \left(\mathbb{E}\left[e^{-\xi Z}\right]\right)\right|=\left|k_{X}(\xi)\right|$ is non-increasing. So

$$
\sup _{\xi>0} \frac{\left|k_{X}(\xi)\right|^{r}}{|\xi|^{r}}=\lim _{\xi \rightarrow 0} \frac{\left|k_{X}(\xi)\right|^{r}}{|\xi|^{r}} .
$$

Thanks to the previous estimate of the Lemma

$$
\frac{\left|k_{Z}(\xi)\right|^{r}}{|\xi|^{r}}=\frac{|\xi \mathbb{E}[Z]+\rho(\xi)|^{r}}{|\xi|^{r}}
$$

with $|\rho(\xi)|^{r} \leq C|\xi|^{2 r}$. This gives (67).

Lemma 17. Let $Z$ be a random variables taking values in $\mathbb{N}$ and $X$ a non-negative real random variable. Assume that $\mathbb{E}\left[X^{r}\right]+\mathbb{E}\left[Z^{r}\right]<+\infty$ for some $r \in(1,2)$. Then there is a positive constant $C=C(X)$ such that, for all $\xi>0$

$$
\left|L_{Z}\left(-k_{X}(\xi)\right)-L_{Z}(\xi \mathbb{E}[X])\right| \xi^{-r} \leq C(X)\left[1+\mathbb{E}\left[Z^{r}\right] .\right.
$$

Proof. Let us define

$$
C(X)=\max \left\{c_{r}\left(\mathbb{E}\left[X^{r}\right]+2 \mathbb{E}[X]^{r}\right)+2^{r-2} \mathbb{E}[X]^{r}, 2^{r+1} \mathbb{E}[X]^{r}\right\} .
$$

If $\xi \geq 1 /(2 \mathbb{E}[X])$, it holds

$$
\left|L_{Z}\left(-k_{X}(\xi)\right)-L_{Z}(\xi \mathbb{E}[X])\right| \xi^{-r} \leq 2^{r+1} \mathbb{E}[X]^{r} \leq C(X) .
$$

If now $\xi<1 /(2 \mathbb{E}[X])$ one obtains

$$
\begin{aligned}
\mid L_{Z}\left(-k_{X}(\xi)\right)- & L_{Z}(\xi \mathbb{E}[X])\left|\xi^{-r} \leq\right| L_{Z}\left(-k_{X}(\xi)\right)-1-k_{X}(\xi) \mathbb{E}[Z] \mid \xi^{-r} \\
& +\left|1-\xi \mathbb{E}[X] \mathbb{E}[Z]-L_{Z}(\mathbb{E}[X] \xi)\right| \xi^{-r}+E[Z]\left|\xi \mathbb{E}[X]+k_{X}(\xi)\right| \xi^{-r} \\
& \leq c_{r} \mathbb{E}\left[Z^{r}\right]\left\{\xi^{-r}\left|k_{X}(\xi)\right|^{r}+\mathbb{E}[X]^{r}\right\}+\mathbb{E}\left[Z^{r}\right]\left\{c_{r} \mathbb{E}\left[X^{r}\right]+\xi^{2-r} \mathbb{E}[X]^{2}\right\} \\
& \leq \mathbb{E}\left[Z^{r}\right]\left[c_{r}\left(\mathbb{E}\left[X^{r}\right]+2 \mathbb{E}[X]^{r}\right)+2^{r-2} \mathbb{E}[X]^{r}\right]
\end{aligned}
$$

Note that the last two inequalities from below have been obtained by using (64) and (66) and (67), respectively.

Lemma 18. Let $C:=\sup _{\xi>0}\left|1-e^{-\xi}\right||\xi|^{-1}<+\infty$, and $B=\sup _{0<\xi \leq \log (2)}|\xi|\left|1-e^{-\xi}\right|^{-1}$. Then, for every $r \geq 1$

$$
d_{r}^{*}(f, g) \leq C^{r} d_{r}(f, g),
$$

and

$$
d_{r}(f, g) \leq \max \left\{2^{r+1}, B^{r} d_{r}^{*}(f, g)\right\} .
$$

Moreover, if $M_{1}(f)=M_{1}(g)$, and $M_{r}(f)$ and $M_{r}(g)$ are finite for some $r \in(1,2]$, then

$$
d_{r}^{*}(f, g) \leq c_{r}\left[M_{r}(f)+M_{r}(g)\right],
$$

for a suitable constant $c_{r}$.

Proof. Noticing that $\hat{f}\left(e^{-\xi}\right)=\tilde{f}(\xi)$, one can write

$$
\frac{|\tilde{f}(\xi)-\tilde{g}(\xi)|}{|\xi|^{r}}=\frac{\left|\hat{f}\left(e^{-\xi}\right)-\hat{g}\left(e^{-\xi}\right)\right|}{\left|1-e^{-\xi}\right|^{r}} \frac{\left|1-e^{-\xi}\right|^{r}}{|\xi|^{r}} .
$$


Hence $d_{r}^{*}(f, g) \leq C^{r} d_{r}(f, g)$. Moreover,

$$
\begin{aligned}
& d_{r}(f, g)=\max \left\{\sup _{s \in(0,1 / 2)} \frac{|\hat{f}(s)-\hat{g}(s)|}{|1-s|^{r}}, \sup _{s \in(1 / 2,1)} \frac{|\hat{f}(s)-\hat{g}(s)|}{|1-s|^{r}}\right\} \\
& \leq \max \left\{2^{r+1}, \sup _{\xi \in(0, \log (2))} \frac{\left|\hat{f}\left(e^{-\xi}\right)-\hat{g}\left(e^{-\xi}\right)\right|}{|\xi|^{r}} \frac{|\xi|^{r}}{\left|1-e^{-\xi}\right|^{r}}\right\} \\
& \leq \max \left\{2^{r+1}, B^{r} d_{r}^{*}(f, g)\right\} \text {. }
\end{aligned}
$$

Thanks to $(63)$

$$
\begin{aligned}
\frac{|\tilde{f}(\xi)-\tilde{g}(\xi)|}{|\xi|^{r}} & =\frac{\left|\int\left(e^{-\xi v}-1+v \xi\right) f(d v)-\int\left(e^{-\xi v}-1+v \xi\right) g(d v)\right|}{|\xi|^{r}} \\
& \leq c_{r}\left[M_{r}(f)+M_{r}(g)\right] .
\end{aligned}
$$

A.3. Moments of random sums. Let $V_{1}$ and $V_{2}$ two integer valued random variables. Let $\left(X_{k}\right)_{k \geq 1}$ and $\left(Y_{k}\right)_{k \geq 1}$ two sequences of iid non-negative random variables and assume that $V_{1}, V_{2}$, $\left(X_{k}\right)_{k \geq 1},\left(Y_{k}\right)_{k \geq 1}$ are independent.

Lemma 19. Under the previous assumptions let $r \in(1,2]$ and assume that $\mathbb{E}\left[Y^{r}+Y^{r}\right]<+\infty$ and $\mathbb{E}\left[V_{1}^{r}+V_{2}^{r}\right]<+\infty$. Then, if $r=2$

$$
\begin{aligned}
\mathbb{E}\left[\left(\sum_{i=1}^{V_{1}} X_{i}+\sum_{i=1}^{V_{2}} Y_{i}\right)^{2}\right] & =\mathbb{E}\left[V_{1}\right] \operatorname{Var}\left(X_{1}\right)+\mathbb{E}\left[V_{2}\right] \operatorname{Var}\left(Y_{1}\right) \\
& +2 \mathbb{E}\left[V_{1}\right] \mathbb{E}\left[V_{2}\right] \mathbb{E}\left[X_{1}\right] \mathbb{E}\left[Y_{2}\right]+\mathbb{E}\left[V_{1}^{2}\right] \mathbb{E}\left[X_{1}\right]^{2}+\mathbb{E}\left[V_{2}^{2}\right] \mathbb{E}\left[Y_{1}\right]^{2},
\end{aligned}
$$

while if $r \in(1,2)$

$$
\begin{aligned}
& \mathbb{E}\left[\left(\sum_{i=1}^{V_{1}} X_{i}+\sum_{i=1}^{V_{2}} Y_{i}\right)^{r}\right] \leq \mathbb{E}\left[V_{1}\right] \mathbb{E}\left[X_{1}^{r}\right]+\mathbb{E}\left[V_{2}\right] \mathbb{E}\left[Y_{1}^{r}\right]+ \\
&\left(2 \mathbb{E}\left[V_{1}\right] \mathbb{E}\left[V_{2}\right] \mathbb{E}\left[X_{1}\right] \mathbb{E}\left[Y_{1}\right]\right)^{\frac{r}{2}}+\mathbb{E}\left[V_{1}^{r}\right] \mathbb{E}\left[X_{1}\right]^{r}+\mathbb{E}\left[V_{2}^{r}\right] \mathbb{E}\left[Y_{2}\right]^{r} .
\end{aligned}
$$

Proof. We only proof (70). Let $n_{1}$ and $n_{2}$ two integer numbers. Since $r / 2<1$, for any given set of positive numbers $z_{1}, \ldots, z_{n},\left(\sum_{i=1}^{n} z_{i}\right)^{r / 2} \leq \sum_{i=1}^{n} z_{i}^{r / 2}$. This implies

$$
\begin{aligned}
& \mathbb{E}\left[\left(\sum_{i=1}^{n_{1}} X_{i}+\sum_{i=1}^{n_{2}} Y_{i}\right)^{2 \frac{r}{2}}\right]=\mathbb{E}\left[\left(\left(\sum_{i=1}^{n_{1}} X_{i}\right)^{2}+\left(\sum_{i=1}^{n_{2}} Y_{i}\right)^{2}+2 \sum_{i=1}^{n_{1}} X_{i} \sum_{i=1}^{n_{2}} Y_{i}\right)^{\frac{r}{2}}\right] \\
& =\mathbb{E}\left[\left(\sum_{i=1}^{n_{1}} X_{i}^{2}+\sum_{i=1}^{n_{2}} Y_{i}^{2}+2 \sum_{i=1}^{n_{1}} X_{i} \sum_{i=1}^{n_{2}} Y_{i}+\sum_{i \neq j, i, j=1}^{n_{1}} X_{i} X_{j}+\sum_{i \neq j, i, j=1}^{n_{2}} Y_{i} Y_{j}\right)^{\frac{r}{2}}\right] \\
& \leq \mathbb{E}\left[\sum_{i=1}^{n_{1}} X_{i}^{r}+\sum_{i=1}^{n_{2}} Y_{i}^{r}+2^{\frac{r}{2}}\left(\sum_{i=1}^{n_{1}} X_{i} \sum_{i=1}^{n_{2}} Y_{i}\right)^{\frac{r}{2}}+\left(\sum_{i \neq j, i, j=1}^{n_{1}} X_{i} X_{j}\right)^{\frac{r}{2}}+\left(\sum_{i \neq j, i, j=1}^{n_{2}} Y_{i} Y_{j}\right)^{\frac{r}{2}}\right] \\
& \leq n_{1} \mathbb{E}\left[X_{1}^{r}\right]+n_{2} \mathbb{E}\left[Y_{1}^{r}\right]+2^{\frac{r}{2}} \mathbb{E}\left[\sum_{i=1}^{n_{1}} X_{i} \sum_{i=1}^{n_{2}} Y_{i}\right]^{\frac{r}{2}}+\mathbb{E}\left[\sum_{i \neq j, i, j=1}^{n_{1}} X_{i} X_{j}\right]^{\frac{r}{2}}+\mathbb{E}\left[\sum_{i \neq j, i, j=1}^{n_{2}} Y_{i} Y_{j}\right]^{\frac{r}{2}} \\
& \leq n_{1} \mathbb{E}\left[X_{1}^{r}\right]+n_{2} \mathbb{E}\left[Y_{1}^{r}\right]+2^{\frac{r}{2}}\left(n_{1} n_{2} \mathbb{E}\left[X_{1}\right] \mathbb{E}\left[Y_{1}\right]\right)^{\frac{r}{2}}+n_{1}^{r} \mathbb{E}\left[X_{1}\right]^{r}+n_{2}^{r} \mathbb{E}\left[Y_{1}\right]^{r} .
\end{aligned}
$$

Note that we used Jensen's inequality to get the last line. To conclude let us apply the previous inequality conditionally on $V_{1}$ and $V_{2}$ to get

$$
\begin{gathered}
\mathbb{E}\left[\left(\sum_{i=1}^{V_{1}} X_{i}+\sum_{i=1}^{V_{2}} Y_{i}\right)^{r}\right] \leq \\
\mathbb{E}\left[V_{1}\right] \mathbb{E}\left[X_{1}^{r}\right]+\mathbb{E}\left[V_{2}\right] \mathbb{E}\left[Y_{1}^{r}\right]+\mathbb{E}\left[V_{1}^{\frac{r}{2}}\right] \mathbb{E}\left[V_{2}^{\frac{r}{2}}\right]\left(2 \mathbb{E}\left[X_{1}\right] \mathbb{E}\left[Y_{1}\right]\right)^{\frac{r}{2}}+ \\
\mathbb{E}\left[V_{1}^{r}\right] \mathbb{E}\left[X_{1}\right]^{r}+\mathbb{E}\left[V_{2}^{r}\right] \mathbb{E}\left[Y_{2}\right]^{r}
\end{gathered}
$$


and then apply the Jensen's inequality one more time to get $\mathbb{E}\left[V_{i}^{\frac{r}{2}}\right] \leq \mathbb{E}\left[V_{i}\right]^{\frac{r}{2}}$.

A.4. Gronwall's inequality. A well-known version of the Gronwall lemma is the following.

Lemma 20. Suppose that $t \mapsto u(t)$ and $t \mapsto h(t)$ are integrable functions on the interval $[0, T]$. If for some constant $C>0$

$$
u(t) \leq h(t)+C \int_{0}^{t} u(s) d s \quad 0 \leq t \leq T
$$

then, for every $t \in[0, T]$

$$
u(t) \leq h(t)+C \int_{0}^{t} e^{C(t-s)} h(s) d s .
$$

A simple consequence of the previous Lemma is the following.

Lemma 21. Let $t \mapsto u(t)$ be a positive measurable function on the interval $[0,+\infty)$ such that:

(i) $I=\{t: u(t)<+\infty\}$ is an open interval $\left(0, s_{0}\right)$,

(ii) $u$ is continuous on $I$

(iii) if $s_{0}<+\infty$ then

$$
\lim _{t \rightarrow s_{0}^{-}} u(s)=+\infty .
$$

If there is a non-negative continuous function $t \mapsto h(t)$ on $[0,+\infty)$ and a constant $C>0$ such that

for every $t \geq 0$, then

$$
u(t) \leq h(t)+C \int_{0}^{t} u(s) d s,
$$

$$
u(t) \leq h(t)+C \int_{0}^{t} e^{C(t-s)} h(s) d s,
$$

for every $t>0$ and, in particular, $s_{0}=\infty$. Finally, if $h(t)$ is of bounded variation then

$$
u(t) \leq e^{C t} h(0)+\int_{0}^{t} e^{C(t-s)} d h(s) .
$$

Proof. It is enough to prove that $s_{0}=+\infty$. Indeed in this case the thesis follows from the previous Lemma. Assume that $s_{0}<+\infty$. Let $H(t)=h(t)+C \int_{0}^{t} e^{C(t-s)} h(s) d s$. By (ii) and the fact that $H(t)$ is bounded on every finite interval, one can choose $\epsilon$ in such a way that $u\left(s_{0}-\epsilon\right)>H\left(s_{0}-\epsilon\right)$. Now $u$ is continuous in $\left(0, s_{0}\right)$ and hence it is bounded on $\left(0, s_{0}-\epsilon\right)$. Hence, by the previous Lemma, $u\left(s_{0}-\epsilon\right) \leq H\left(s_{0}-\epsilon\right)$ which gives a contradiction. The last part of the proof follows resorting to integration by parts.

ACKNowledgements: This work has been written within the activities of GNFM (G.T.) and GNAMPA (F.B.) groups of INdAM (National Institute of High Mathematics), and partially supported by MIUR project "Optimal mass transportation, geometrical and functional inequalities with applications" (G.T.).

\section{REFERENCES}

[1] Gerold Alsmeyer and Matthias Meiners. Fixed points of the smoothing transform: two-sided solutions. Probab. Theory Related Fields, 155(1-2):165-199, 2013.

[2] F. Bassetti and L. Ladelli. Self-similar solutions in one-dimensional kinetic models: a probabilistic view. Ann. Appl. Probab., 22(5):1928-1961, 2012.

[3] F. Bassetti, L. Ladelli, and D. Matthes. Central limit theorem for a class of one-dimensional kinetic equations. Probab. Theory Related Fields, 150(1-2):77-109, 2011.

[4] F. Bassetti and E. Perversi. Speed of convergence to equilibrium in Wasserstein metrics for Kac-like kinetic equations. Electron. J. Probab., 18:no. 6, 35, 2013.

[5] F. Bassetti and G. Toscani. Explicit equilibria in a kinetic model of gambling. Phys. Rev. E (3), 81(6):066115, $7,2010$. 
[6] F. Bassetti and G. Toscani. Explicit equilibria in bilinear kinetic models for socio-economic interactions. ESAIM: Proc. and Surveys, 47:1-16, 2014.

[7] F. Baumdicker, W. R. Hess, and P. Pfaffelhuber. The diversity of a distributed genome in bacterial populations. Ann. Appl. Probab., 20(5):1567-1606, 2010.

[8] N. Bellomo. Modeling complex living systems. Modeling and Simulation in Science, Engineering and Technology. Birkhäuser Boston, Inc., Boston, MA, 2008. A kinetic theory and stochastic game approach.

[9] N. Bellomo, A. Bellouquid, and M. Delitala. Mathematical topics on the modelling complex multicellular systems and tumor immune cells competition. Math. Models Methods Appl. Sci., 14(11):1683-1733, 2004.

[10] N. Bellomo and M. Delitala. From the mathematical kinetic, and stochastic game theory to modelling mutations, onset, progression and immune competition of cancer cells. Physics of Life Reviews, 5(4):183-206, 2008 .

[11] N. Bellomo, N. K. Li, and P. K. Maini. On the foundations of cancer modelling: selected topics, speculations, and perspectives. Math. Models Methods Appl. Sci., 18(4):593-646, 2008.

[12] P. Billingsley. Probability and measure. Wiley Series in Probability and Mathematical Statistics. John Wiley \& Sons Inc., New York, third edition, 1995. A Wiley-Interscience Publication.

[13] A. V. Bobylëv. The method of the Fourier transform in the theory of the Boltzmann equation for Maxwell molecules. Dokl. Akad. Nauk SSSR, 225(6):1041-1044, 1975.

[14] A.V. Bobylev, C. Cercignani, and I.M. Gamba. On the self-similar asymptotics for generalized nonlinear kinetic Maxwell models. Comm. Math. Phys., 291(3):599-644, 2009.

[15] Leo Breiman. Probability, volume 7 of Classics in Applied Mathematics. Society for Industrial and Applied Mathematics (SIAM), Philadelphia, PA, 1992. Corrected reprint of the 1968 original.

[16] M. Burger, L. Caffarelli, P. A. Markowich, and M-T. Wolfram. On a Boltzmann-type price formation model. Proc. R. Soc. Lond. Ser. A Math. Phys. Eng. Sci., 469(2157):20130126, 20, 2013.

[17] M. Burger, L. Caffarelli, P. A. Markowich, and M-T. Wolfram. On the asymptotic behavior of a Boltzmann-type price formation model. Commun. Math. Sci., 12(7):1353-1361, 2014.

[18] M.J. Cáceres and G. Toscani. Kinetic approach to long time behavior of linearized fast diffusion equations. $J$. Stat. Phys., 128(4):883-925, 2007.

[19] J. A. Carrillo, M. Fornasier, J. Rosado, and G. Toscani. Asymptotic flocking dynamics for the kinetic CuckerSmale model. SIAM J. Math. Anal., 42(1):218-236, 2010.

[20] José A. Carrillo, Massimo Fornasier, Giuseppe Toscani, and Francesco Vecil. Particle, kinetic, and hydrodynamic models of swarming. In Mathematical modeling of collective behavior in socio-economic and life sciences, Model. Simul. Sci. Eng. Technol., pages 297-336. Birkhäuser Boston, Inc., Boston, MA, 2010.

[21] Stephane Cordier, Lorenzo Pareschi, and Giuseppe Toscani. On a kinetic model for a simple market economy. J. Stat. Phys., 120(1-2):253-277, 2005.

[22] F. Cucker and E. Mordecki. Flocking in noisy environments. J. Math. Pures Appl. (9), 89(3):278-296, 2008.

[23] F. Cucker and S. Smale. Emergent behavior in flocks. IEEE Trans. Automat. Control, 52(5):852-862, 2007.

[24] F. Cucker and S. Smale. On the mathematics of emergence. Jpn. J. Math., 2(1):197-227, 2007.

[25] R. J. DiPerna and P.-L. Lions. Ordinary differential equations, transport theory and Sobolev spaces. Invent. Math., 98(3):511-547, 1989.

[26] B. Düring, P. Markowich, J-F. Pietschmann, and M-T. Wolfram. Boltzmann and Fokker-Planck equations modelling opinion formation in the presence of strong leaders. Proc. R. Soc. Lond. Ser. A Math. Phys. Eng. Sci., 465(2112):3687-3708, 2009.

[27] W. Feller. An introduction to probability theory and its applications. Vol. I. Third edition. John Wiley \& Sons Inc., New York, 1968.

[28] G. Furioli, A. Pulvirenti, E. Terraneo, and G. Toscani. The grazing collision limit of the inelastic Kac model around a Lévy-type equilibrium. SIAM J. Math. Anal., 44(2):827-850, 2012.

[29] G. Gabetta, G. Toscani, and B. Wennberg. Metrics for probability distributions and the trend to equilibrium for solutions of the Boltzmann equation. J. Statist. Phys., 81(5-6):901-934, 1995.

[30] I. S. Gradshteyn and I. M. Ryzhik. Table of integrals, series, and products. Elsevier/Academic Press, Amsterdam, seventh edition, 2007. Translated from the Russian, Translation edited and with a preface by Alan Jeffrey and Daniel Zwillinger, With one CD-ROM (Windows, Macintosh and UNIX).

[31] J. Grilli, M. Romano, F. Bassetti, and Cosentino Lagomarsino M. Cross-species gene-family fluctuations reveal the dynamics of horizontal transfers. Nucleic Acids Research, 42(11), 2014.

[32] S-Y. Ha and E. Tadmor. From particle to kinetic and hydrodynamic descriptions of flocking. Kinet. Relat. Models, 1(3):415-435, 2008.

[33] E. Kashdan and L. Pareschi. Mean field mutation dynamics and the continuous Luria-Delbrück distribution. Math. Biosci., 240(2):223-230, 2012.

[34] E. V. Koonin, K.S. Makarova, and Aravind L. Horizontal gene transfer in prokaryotes: quantification and classification. Annu. Rev. Microbiol., 55:709-742, 2001.

[35] E. V. Koonin and Y.I. Wolf. Genomics of bacteria and archaea: the emerging dynamic view of prokaryotic world. Nucleic Acids Res., 36:6688-6719, 2008.

[36] D.E. Lea and C.A. Coulson. The distribution of the numbers of mutants in bacterial populations. Journal of genetics, 1949. 
[37] Quansheng Liu. Fixed points of a generalized smoothing transformation and applications to the branching random walk. Adv. in Appl. Probab., 30(1):85-112, 1998.

[38] S.E. Luria and M. Delbrück. Interference between inactivated bacterial virus and active virus of the same strain and of a different strain. Arch. Biochem, 1942.

[39] S.E. Luria and M. Delbrück. Mutations of bacteria from virus sensitivity to virus resistance. Genetics, 1943.

[40] D. Matthes and G. Toscani. On steady distributions of kinetic models of conservative economies. J. Stat. Phys., 130(6):1087-1117, 2008.

[41] G. Naldi, L. Pareschi, and G. Toscani. Mathematical modeling of collective behavior in socio-economic and life sciences. Springer Verlag, Heidelberg, 2010.

[42] L. Pareschi and G. Toscani. Self-similarity and power-like tails in nonconservative kinetic models. J. Stat. Phys., 124(2-4):747-779, 2006.

[43] L. Pareschi and G. Toscani. Interacting multiagent systems. Kinetic equations \&s Monte Carlo methods. Oxford University Press, Oxford, 2013.

[44] E. Perversi and E. Regazzini. Weak convergence of probability-valued solutions of general one-dimensional kinetic equations: Characterizations and applications. J. Stat. Phys., +2015. to appear, arXiv:1404.6124.

[45] F.W. Steutel and K. van Harn. Infinite divisibility of probability distributions on the real line, volume 259 of Monographs and Textbooks in Pure and Applied Mathematics. Marcel Dekker Inc., New York, 2004.

[46] G. Toscani. Kinetic models of opinion formation. Commun. Math. Sci., 4(3):481-496, 2006.

[47] G. Toscani. A kinetic description of mutation processes in bacteria. Kinet. Relat. Models, 6(4):1043-1055, 2013.

[48] C. Villani. On a new class of weak solutions to the spatially homogeneous Boltzmann and Landau equations. Arch. Rational Mech. Anal., 143(3):273-307, 1998.

[49] E. Wild. On Boltzmann's equation in the kinetic theory of gases. Proc. Cambridge Philos. Soc., 47:602-609, 1951.

[50] Qi Zheng. Progress of a half century in the study of the Luria-Delbrück distribution. Math. Biosci., 162(12):1-32, 1999. 